\title{
Parameterized Reduced Order Models from a Single Mesh Using Hyper-Dual Numbers
}

\author{
M. R. W. Brake ${ }^{\mathrm{a}, *}$, J. A. Fike ${ }^{\mathrm{a}}$, S. D. Topping ${ }^{\mathrm{b}}$ \\ ${ }^{a}$ Sandia National Laboratories, Albuquerque, NM 87185, USA \\ ${ }^{b}$ University of Arizona, Tucson, AZ 85721, USA
}

\begin{abstract}
In order to assess the predicted performance of a manufactured system, analysts must consider random variations (both geometric and material) in the development of a model, instead of a single deterministic model of an idealized geometry with idealized material properties. The incorporation of random geometric variations, however, potentially could necessitate the development of thousands of nearly identical solid geometries that must be meshed and separately analyzed, which would require an impractical number of man-hours to complete. This research advances a recent approach to uncertainty quantification by developing parameterized reduced order models. These parameterizations are based upon Taylor series expansions of the system's matrices about the ideal geometry, and a component mode synthesis representation for each linear substructure is used to form an efficient basis with which to study the system. The numerical derivatives required for the Taylor series expansions are obtained via hyper-dual numbers, and are compared to parameterized models constructed with finite difference formulations. The advantage of using hyper-dual numbers is two-fold: accuracy of the derivatives to machine precision, and the need to only generate a single mesh of the system of interest. The theory is applied to a stepped beam system in order to demonstrate proof of concept. The results demonstrate that the hyper-dual number multivariate parameterization of geometric variations, which largely are neglected in the literature, are accurate for both sensitivity and optimization studies. As model and mesh generation can constitute the greatest expense of time in analyzing a system, the foundation to create a parameterized reduced order model based off of a single mesh is expected to reduce dramatically the necessary time to
\end{abstract}

\footnotetext{
${ }^{*}$ Corresponding author; mrbrake@sandia.gov

${ }^{1}$ Sandia National Laboratories is a multi-program laboratory managed and operated by Sandia Corporation, a wholly owned subsidiary of Lockheed Martin Corporations, for the U.S. Department of Energy's National Nuclear Security Administration under Contract DE-AC04-94AL85000.
} 
analyze multiple realizations of a component's possible geometry.

Keywords: Model Reduction Theory, Parameterization, Uncertainty, Parameterized Reduced Order Models, Hyper-Dual Numbers

\section{Introduction}

Modern engineering analysis must take into account the effects of aleatoric (parametric) uncertainty in the analysis of a system. As a real system is manufactured, part-to-part variations are introduced that can have significant ramifications on the functionality of the system. Thus, in order to account for these variations at the design stage, a methodology is needed to assess the performance of many (often thousands) of permutations of a design to qualify the expected performance of a manufactured system.

One method to simulate the performance of a system is via high fidelity modeling, such as using the finite element (FE) method. High fidelity computational simulations often can provide very accurate predictions; however, they have a very high computational cost. In order to develop simulations that are both efficient and accurate enough, reduced order models (ROMs) often are used as surrogates for a full order model in order to decrease the computational expense of analysis.

To model the perturbations that are found in manufactured systems without a systematic, efficient reduced order approach would be prohibitively difficult. For example, consider a scenario where it takes several weeks to develop a high quality mesh for a representative component, the number of perturbations needed to quantify geometric variation is estimated to be in the thousands ${ }^{2}$, and each likely requires a new mesh. Even with factoring in time saved from some automation of the process, the number of man hours required to construct these meshes is on the order of decades. In addition, if each model takes even two hours to solve then the computation time can be estimated to be on the order of years. Clearly, decades of time are infeasible constraints to be incorporated into a design cycle. One method of accounting for these perturbations is to create a parameterized

\footnotetext{
${ }^{2}$ For a device that has a dozen design features of interest, to statistically sample the realistic range of possible permutations requires analysis of over one thousand variations due to potential nonlinear interactions between the design features and the need to adequately sample the tails of the distribution. New methods such as stochastic reduced order models (Field et al., 2015; Mignolet and Soize, 2008), which determine the most representative samples to use for a distribution if computational limitations restrict the number of simulations that can be performed, can significantly reduce the number of possible permutations, and are anticipated to be a powerful tool when combined with PROMs.
} 
reduced order model (PROM) of the system (Hong et al., 2012, 2013; Kammer and Nimityongskul, 2011; Kammer and Krattiger, 2013; Momin et al., 2010; Voorhees et al., 2011). This allows the behavior of the system to be predicted inexpensively over a range of perturbations based on a set of simulations distributed about the nominal design.

A standard approach to constructing ROMs for structural dynamics is Craig-Bampton (C-B) Component Mode Synthesis (CMS) (Craig and Bampton, 1968). This modeling approach is based on the principles of linear structural dynamics. Nonlinear ROMs exist, such as for the von Karman plate (Brake and Segalman, 2010; Brake et al., 2012) or structures with discrete nonlinearities (Brake and Segalman, 2013), that are amenable for developing parameterized models of nonlinear systems (however, the present work is restricted to linear models). In general, many methods exist to account for the variability due to parameterizations of linear model properties (Xia and Tang, 2013), such as the elastic modulus; however, incorporation of variations of geometric properties (such as a flange clearance), or other properties that are nonlinearly related to the outputs of interest (e.g. natural frequencies), into a parameterized model are much more difficult to achieve. Alternative methods are available, though, such as the stochastic finite element method (Stefanou, 2009), the introduction of random matrices (Grigoriu, 2009), or a surrogate modeling approach ${ }^{3}$ that reduces possible parameterizations to well characterized functions acting upon a significantly reduced system (Brake et al., 2011). In what follows, the present research is developed for a linear vibration application; however, the theory is not limited to linear models.

\subsection{Considerations for the Development of Parameterized Reduced Order Models}

Figure 1 shows two approaches that can be taken to analyze a system composed of several components. The left branch of the diagram shows the traditional approach of forming a full order FE model for the system, which often is prohibitively expensive for assessing aleatoric uncertainty. The right branch shows the steps when C-B CMS is used. To account for aleatoric uncertainty, parameterized ROMs (PROMs) can be utilized. PROMs can be constructed from a finite approximation; for instance, in computing some scalar quantity of interest $f(x)$ as a function of $x=x_{0}+\triangle x$, which is composed of a perturbation $\triangle x$ and the nominal design $x_{0}, f(x)$ can be approximated as

\footnotetext{
${ }^{3}$ An example of a surrogate model might be a single degree of freedom system in which the influence of a design variable is represented as a function, for example: the geometrical and material parameters of contact pins being represented as a contact force function that takes as arguments the physical description of the contact pins. This approach allows for a model in which the potential system perturbations are all relegated to deterministic functions.
} 
$\bar{f}(x)$, which is based on a Taylor series expansion. For the FE and CMS cases outlined in Fig. 1, $f(x)$ could be elements of the mass and stiffness matrices or it could be the result of the system analysis, such as displacements or eigenvalues. The perturbations to the nominal design $\Delta x$ could be changes in material properties or geometric variations. A parameterized model can then be expressed as

$$
\bar{f}\left(x_{0}+\triangle x\right)=f(x)+(\triangle x) f^{\prime}\left(x_{0}\right)+\frac{(\triangle x)^{2}}{2 !} f^{\prime \prime}\left(x_{0}\right)+\frac{(\triangle x)^{3}}{3 !} f^{\prime \prime \prime}\left(x_{0}\right)+\ldots
$$

for a single variation. In practice, this infinite sum is truncated at a finite number of terms. In what follows, second, third, and fourth order expansions are considered.

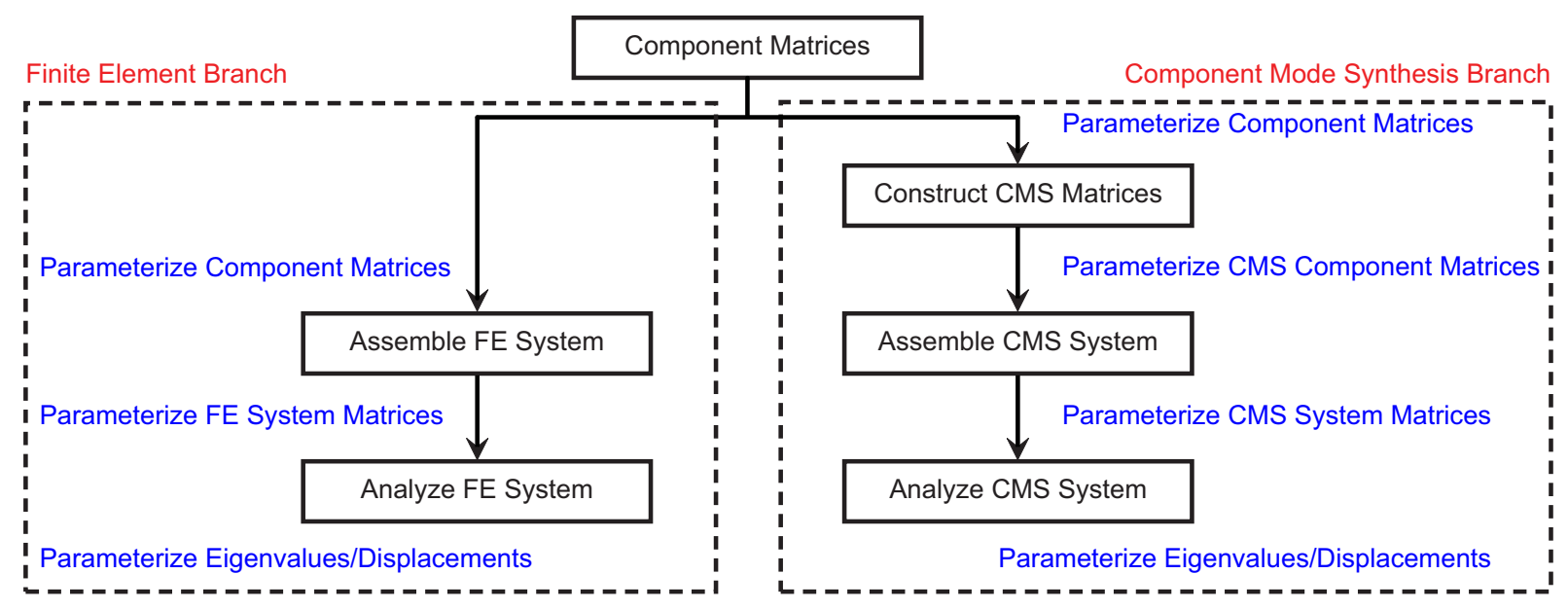

Figure 1: Two possible approaches for analyzing a system composed of several components, and the levels at which parameterized models can be constructed.

The use of these parameterized models with FE analysis requires the calculation of derivatives evaluated at the nominal design, as expressed in Eq. 1. The calculation of these derivatives is the challenge in constructing a PROM. These derivatives thus can be computed via multiple methods. In the present work, they are calculated two different ways: via finite difference approximations, as discussed in $\S 3$, and via hyper-dual numbers, as discussed in $\S 4$. There are numerous advantages and disadvantages with each of these approaches, as discussed throughout the rest of this paper.

Finite difference approximations can require multiple meshes for each dimension of a perturbation, which can result in an impractically large amount of man-hours due to meshing of models. For instance, a fourth order accurate cubic finite difference expansion for one variable would require seven meshes, two variables would require 49 separate meshes, three variables would require 343 
meshes, and $N$ variables would require $7^{N}$ meshes. For a discussion of the derivation of the number of meshes needed for a multivariate finite difference expansion, refer to (Sauer, 1995).

The second approach proposed here is to compute the derivatives using hyper-dual numbers (Fike and Alonso, 2011). Derivatives for the system are calculated via the complex step expansion theorem, which is detailed in $\S 4$. The advantage of using a hyper-dual number representation is that it enables calculations of the exact values of the derivatives. The application of the complexstep approach to ordinary complex numbers, by contrast, is subject to truncation error (for all derivatives) and subtractive cancelation error (for second or higher derivatives) as discussed in the next sections. The use of hyper-dual numbers only requires the nominal mesh (in contrast to finite difference-based approaches, as discussed in $\S 4$ ), but the information on how the mesh would change due to the perturbations needs to be known or calculated. In practice, this can require the introduction of hyper-dual numbers into the meshing procedure.

Parameterized models can be created at several levels in the analysis procedure outlined in Fig. 1. One possibility is to construct parameterized models for the component mass and stiffness matrices. Another possibility is to parameterize the output of the system analysis, i.e. displacements or eigenvalues. When using CMS it is also possible to parameterize the reduced order mass and stiffness matrices. In each of these levels, the hyper-dual numbers are introduced at the construction of the FE matrices. One goal of this research is to assess the efficacy of developing PROMs at each level identified in Fig. 1. Specifically, this research seeks to answer several questions:

- At what level should a parameterization be conducted?

- What order parameterization is necessary?

- How many meshes are required to develop an accurate parameterization?

- What is the domain/extent of validity of the parameterization?

To answer these questions, this paper first presents a brief overview of C-B CMS in $\S 2$, and the development of PROMs in $\S 3$. Hyper-dual numbers are introduced, and the development of PROMs based on hyper-dual numbers are discussed in $\S 4$. Next, $\S 5$ assesses the efficacy of each potential level (as indicated in Fig. 1) of developing a single variable PROM using both finite difference based approaches and hyper-dual number based approaches, and $\S 6$ assesses the efficacy of multivariate PROMs. The analysis of $\S 5$ is a structural dynamics problem chosen to illustrate 
the present methods; the mathematics behind the approach, though, are applicable to a more generalized parameterized model development framework. Finally, conclusions and answers to the questions posed above are presented in $\S 7$.

\section{Craig-Bampton Component Mode Synthesis}

The equation of motion for an unforced and undamped linear structure with mass matrix $\mathbf{M}$, stiffness matrix $\mathbf{K}$, and displacement vector $\mathbf{u}$ is

$$
\mathbf{M u ̈}+\mathbf{K u}=\mathbf{0} .
$$

The C-B CMS method (Craig and Bampton, 1968) is based on a restructuring of each substructure's degrees of freedom (DOFs) into boundary DOFs $\mathbf{u}_{b}$ and internal DOFs $\mathbf{u}_{i}$ as $\mathbf{u}=\left\{\mathbf{u}_{b}^{T}, \mathbf{u}_{i}^{T}\right\}^{T}$. Boundary DOFs typically are defined as DOFs where excitations are applied or where output quantities are desired (such as the displacement of a particular flange), whereas internal DOFs are all non-boundary DOFs, which generally are inside of the component and thus have no applied loads. This substructuring is used to recast Eq. 2 as

$$
\left[\begin{array}{ll}
\mathbf{M}_{b b} & \mathbf{M}_{b i} \\
\mathbf{M}_{i b} & \mathbf{M}_{i i}
\end{array}\right]\left\{\begin{array}{l}
\ddot{\mathbf{u}}_{b} \\
\ddot{\mathbf{u}}_{i}
\end{array}\right\}+\left[\begin{array}{ll}
\mathbf{K}_{b b} & \mathbf{K}_{b i} \\
\mathbf{K}_{i b} & \mathbf{K}_{i i}
\end{array}\right]\left\{\begin{array}{l}
\mathbf{u}_{b} \\
\mathbf{u}_{i}
\end{array}\right\}=\mathbf{0} .
$$

Modal analysis is performed on the internal DOFs

$$
\left(\mathbf{K}_{i i}-\lambda_{\ell} \mathbf{M}_{i i}\right) \phi_{\ell}=0
$$

yielding eigenvalues $\lambda_{\ell}$ and corresponding eigenvectors $\phi_{\ell}$ of the fixed interface normal modes. The constraint modes, $\boldsymbol{\Phi}_{C}$, are static deflection shapes and are computed by imposing a unit displacement at each individual boundary DOF while holding the other boundary DOFs fixed at zero displacement. The constraint modes can therefore be computed as

$$
\mathbf{\Phi}_{C}=-\mathbf{K}_{i i}^{-1} \mathbf{K}_{i b}
$$

Subsequently, the physical DOFs $\mathbf{u}_{b}$ and $\mathbf{u}_{i}$ are related to the hybrid set of physical and modal DOFs $\mathbf{u}_{b}$ and $\mathbf{q}_{m}$ by

$$
\left\{\begin{array}{c}
\mathbf{u}_{b} \\
\mathbf{u}_{i}
\end{array}\right\}=\left[\begin{array}{cc}
\mathbf{I} & \mathbf{0} \\
\boldsymbol{\Phi}_{C} & \boldsymbol{\Phi}_{N}
\end{array}\right]\left\{\begin{array}{c}
\mathbf{u}_{b} \\
\mathbf{q}_{m}
\end{array}\right\}
$$


with the identity matrix $\mathbf{I}$ and where $\boldsymbol{\Phi}_{N}$ is a matrix of the eigenvectors $\boldsymbol{\phi}_{\ell}$. Substitution yields the equation of motion for each component to be written as (Young, 2000)

$$
\left[\begin{array}{cc}
\mathbf{M}_{C C} & \mathbf{M}_{C N} \\
\mathbf{M}_{N C} & \mathbf{I}
\end{array}\right]\left\{\begin{array}{c}
\ddot{\mathbf{u}}_{b} \\
\ddot{\mathbf{q}}_{m}
\end{array}\right\}+\left[\begin{array}{cc}
\mathbf{K}_{C C} & \mathbf{0} \\
\mathbf{0} & \mathbf{\Lambda}^{2}
\end{array}\right]\left\{\begin{array}{c}
\mathbf{u}_{b} \\
\mathbf{q}_{m}
\end{array}\right\}=\{0\},
$$

in which the eigenvectors are normalized with respect to the mass matrix $\mathbf{M}_{i i}$, such that

$$
\begin{gathered}
\mathbf{M}_{C C}=\mathbf{M}_{b b}+\mathbf{M}_{b i} \boldsymbol{\Phi}_{C}+\boldsymbol{\Phi}_{C}^{T} \mathbf{M}_{i b}+\boldsymbol{\Phi}_{C}^{T} \mathbf{M}_{i i} \boldsymbol{\Phi}_{C} \\
\mathbf{M}_{C N}=\mathbf{M}_{N C}^{T}=\left(\mathbf{M}_{b i}+\boldsymbol{\Phi}_{C}^{T} \mathbf{M}_{i i}\right) \boldsymbol{\Phi}_{N} \\
\mathbf{K}_{C C}=\mathbf{K}_{b b}+\mathbf{K}_{b i} \boldsymbol{\Phi}_{C}+\boldsymbol{\Phi}_{C}^{T}\left(\mathbf{K}_{i b}+\mathbf{K}_{i i} \boldsymbol{\Phi}_{C}\right)=\mathbf{K}_{b b}+\mathbf{K}_{b i} \boldsymbol{\Phi}_{C}
\end{gathered}
$$

and where $\Lambda^{2}$ is a diagonal matrix of the squares of the eigenvalues $\lambda_{\ell}^{2}$.

The number of DOFs can be reduced by retaining only enough fixed interface normal modes to capture the behavior of interest. In general, keeping more modes results in a more accurate approximation of the true behavior at the expense of an increased computational cost due to the increase in number of DOFs retained. The reduced mass and stiffness matrices for each component can be combined to form a ROM for the system. This ROM is able to provide accurate results if enough modes are kept to capture the behavior of interest.

\section{Finite Difference Parameterizations}

In other PROM formulations, such as (Hong et al., 2013), the parameterizations of the CMS matrices are calculated using Taylor series expansions in which the derivatives are calculated numerically using a finite difference scheme. Given a system matrix $\mathbf{A}(x)$ defined for a parameter $x=x_{0}+\triangle x$ having a nominal value $x_{0}$ and a variation $\triangle x$, the parameterized system matrix is

$$
\mathbb{A}(x)=\mathbf{A}\left(x_{0}\right)+(\triangle x) \mathbf{A}^{\prime}\left(x_{0}\right)+\frac{(\triangle x)^{2}}{2} \mathbf{A}^{\prime \prime}\left(x_{0}\right)+\frac{(\triangle x)^{3}}{3 !} \mathbf{A}^{\prime \prime \prime}\left(x_{0}\right)+\mathcal{O}\left((\triangle x)^{4}\right)
$$

from the Taylor series expansion. The derivatives $\mathbf{A}^{\prime}, \mathbf{A}^{\prime \prime}$, and higher order terms, are calculated via finite difference approximations. For an approximation of second order accuracy, the first and second derivatives are (Hughes, 2000)

$$
\mathbf{A}^{\prime}\left(x_{0}\right)=\frac{\mathbf{A}\left(x_{0}+h\right)-\mathbf{A}\left(x_{0}-h\right)}{2 h}
$$




$$
\mathbf{A}^{\prime \prime}\left(x_{0}\right)=\frac{\mathbf{A}\left(x_{0}+h\right)-2 \mathbf{A}\left(x_{0}\right)+\mathbf{A}\left(x_{0}-h\right)}{h^{2}}
$$

for a small perturbation $h$. For higher order approximations, considerably more terms are required, as seen for the approximations of the first and second derivatives with fourth order accuracy

$$
\begin{gathered}
\mathbf{A}^{\prime}\left(x_{0}\right)=\frac{-\mathbf{A}\left(x_{0}+2 h\right)+8 \mathbf{A}\left(x_{0}+h\right)-8 \mathbf{A}\left(x_{0}-h\right)+\mathbf{A}\left(x_{0}-2 h\right)}{12 h} \\
\mathbf{A}^{\prime \prime}\left(x_{0}\right)=\frac{-\mathbf{A}\left(x_{0}+2 h\right)+16 \mathbf{A}\left(x_{0}+h\right)-30 \mathbf{A}\left(x_{0}\right)+16 \mathbf{A}\left(x_{0}-h\right)-\mathbf{A}\left(x_{0}-2 h\right)}{h^{2}} .
\end{gathered}
$$

Equation 11 has error on the order of $(\triangle x)^{4}$, as noted by the term $\mathcal{O}\left((\triangle x)^{4}\right)$. In general, for an accuracy of $N, N$ evaluations of $\mathbf{A}$ are needed for a first derivative, $N+1$ evaluations of $\mathbf{A}$ are needed for a second derivative, $N+2$ evaluations for a third derivative, and $N+j-1$ evaluations for a $j^{\text {th }}$ derivative. In practice, due to the overlap between the evaluations needed for each derivative (such as $\mathbf{A}(x+h)$ in both (12) and (13)), $N+j$ evaluations of $\mathbf{A}$ are needed for a parameterization that includes up to $j^{\text {th }}$ order derivatives of accuracy $N$ for a single-variate expansion. Note that for each evaluation in Eqs. 12-15, a new mesh of the system is required when $x$ is a geometric parameter.

For a multivariate expansion, the number of evaluations of $\mathbf{A}$ geometrically increases, such that a second order accurate expansion of a five parameter problem $\mathbf{x}=\left\{x_{1}, x_{2}, x_{3}, x_{4}, x_{5}\right\}$ requires 51 evaluations of $\mathbf{A}$, and a fourth order accurate expansion of the same system requires 301 evaluations of $\mathbf{A}$. The parameterization of multivariate expansions is further discussed in $\S 6$.

\section{Hyper-Dual Number Parameterizations}

The PROM method proposed in the present work is derived using hyper-dual numbers (Fike and Alonso, 2011), which are based on a branch of complex number theory. A similar approach based on ordinary complex numbers (Voorhees et al., 2011) has shown some success already. The inclusion of hyper-dual numbers into the model development process allows for the exact calculations of derivatives (as opposed to approximations from using ordinary complex numbers, which are subject to numerical error, as discussed in the following text and in Fig. 2) that are necessary in Eq. 11 without needing multiple points at which the derivatives are evaluated, as is discussed in $\S 4.1$. Due to this, the hyper-dual number formulation is a local method as it cannot account for changes in the system away from the nominal geometry (such as two flanges coming into contact/merging if the width of one of them is increased sufficiently). This is in contrast to the finite difference 
approach, which is based on multiple meshes to provide a more global assessment of the system. As model and mesh generation can constitute the greatest expense of time in analyzing a system, the foundation to create a PROM based off of a single mesh is expected to reduce dramatically the necessary time to analyze multiple realizations of a component's possible geometry.

\subsection{Dual Numbers, Hyper-Dual Numbers, and the Complex Step Derivative Calculation}

A dual number (Study, 1903; Karzel and Kist, 1985) is defined as a class of generalized complex numbers where the non-real part is defined by the non-zero root of the number zero $\left(\epsilon^{2}=0\right.$, $\epsilon \neq 0$ ), as described in (Fike and Alonso, 2011). Dual numbers originated in using quaternions as an extension of complex numbers to study geometry and kinematics (Study, 1903). More recently, (Karzel and Kist, 1985) used Study's quaternions to apply kinematic algebra to applications in geometry.

A hyper-dual number is a dual number defined in more than one dimension. For example, two dimensional hyper-dual numbers are defined to consist of one real part and three non-real parts, where the three non-real units $\epsilon_{1}, \epsilon_{2}$, and $\epsilon_{1} \epsilon_{2}$ have the properties that $\epsilon_{1}^{2}=\epsilon_{2}^{2}=\left(\epsilon_{1} \epsilon_{2}\right)^{2}=0$ but $\epsilon_{1} \neq \epsilon_{2} \neq \epsilon_{1} \epsilon_{2} \neq 0$. Higher dimensional hyper-dual numbers can also be considered, such as described later in the text.

Given a generalized complex number E, the complex step derivative calculation method (Abreu et al., 2013; Voorhees et al., 2011) is defined from the Taylor series of a function $f(x)$ expanded about a step $h \mathrm{E}$, where $h$ typically is a small, real-valued number

$$
f(x+h \mathrm{E})=f(x)+h f^{\prime}(x) \mathrm{E}+\frac{1}{2 !} h^{2} \mathrm{E}^{2} f^{\prime \prime}(x)+\frac{1}{3 !} h^{3} \mathrm{E}^{3} f^{\prime \prime \prime}(x)+\cdots
$$

For ordinary complex numbers, $\mathrm{E}^{2}=\mathrm{i}^{2}=-1$, which results in the estimate of the first derivative of $f(x)$ as

$$
f^{\prime}(x) \approx \frac{\operatorname{Imag}[f(x+h \mathrm{i})]}{h}+\mathcal{O}\left(h^{2}\right)
$$

with error $\mathcal{O}\left(h^{2}\right)$ due to the terms $-h^{3} \mathrm{i} f^{\prime \prime \prime}(x) / 3 !+h^{5} \mathrm{i} f^{\prime \prime \prime \prime \prime}(x) / 5 !+\ldots$ Higher order terms could be used with multiple complex step evaluations, but the truncation error would still be present (Lai and Crassidis, 2008). The even terms, $-h^{2} f^{\prime \prime}(x) / 2 !+\ldots$, are purely real as $\mathrm{i}^{2}=-1$. For a dual number, however, $\mathrm{E}^{2}=\epsilon^{2}=0$, resulting in

$$
f(x+h \epsilon)=f(x)+h f^{\prime}(x) \epsilon,
$$


which yields the exact first derivative as the non-real part of the expansion

$$
f^{\prime}(x)=\frac{\operatorname{Dual}[f(x+h \epsilon)]}{h} .
$$

The function Dual $[f(x+h \epsilon)]$ returns the non-real (dual) value of the expression $f(x+h \epsilon)$. Equation 19 is exact mathematically since there are no higher order terms as $\epsilon^{2}=0$. Likewise, the Taylor series for a real-valued function subjected to a hyper-dual perturbation truncates exactly at the second derivative term

$$
f\left(x+h_{1} \epsilon_{1}+h_{2} \epsilon_{2}+0 \epsilon_{1} \epsilon_{2}\right)=f(x)+h_{1} f^{\prime}(x) \epsilon_{1}+h_{2} f^{\prime}(x) \epsilon_{2}+h_{1} h_{2} f^{\prime \prime}(x) \epsilon_{1} \epsilon_{2}
$$

for arbitrary perturbations $h_{1}$ and $h_{2}$. There is no truncation error because all of the higher order terms contain $\epsilon_{1}^{2}$ or $\epsilon_{2}^{2}$ or higher powers and are zero by definition. The first and second derivatives are the leading terms of the non-real parts, and these terms can be found by taking the individual non-real parts and dividing by the step size. There is no required difference operation, as in finite difference approximations, which would lead to subtractive cancelation error. The first and second derivatives can thus be computed exactly, regardless of the step size (Fike et al., 2011).

As an example of the complex step expansion, consider

$$
f(x)=x^{2},
$$

which has the derivative

$$
f^{\prime}(x)=2 x
$$

The complex step expansion, using dual numbers, yields

$$
f(x+\epsilon h)=(x+\epsilon h)^{2}=x^{2}+2 x \epsilon h+\epsilon^{2} h^{2} .
$$

This has a real part

$$
\operatorname{Real}[f(x+h \epsilon)]=x^{2}+\epsilon^{2} h^{2}=x^{2},
$$

and a non-real part

$$
\text { Dual }[f(x+h \epsilon)]=2 x \epsilon h=f^{\prime}(x) \epsilon h .
$$

This mathematical property holds true for any function $f(x)$. To further illustrate the accuracy of 
the hyper-dual number approach, the derivatives of

$$
f(x)=\frac{e^{x}}{\sqrt{(\sin (x))^{3}+(\cos (x))^{3}}} .
$$

are calculated, and the error in the derivative calculations compared to the exact solution are shown in Fig. 2 for a forward difference scheme, a complex step approximation, and hyper-dual numbers. For both the first (Fig. 2(a)) and second (Fig. 2(b)) derivatives, the hyper-dual number method is limited only by the machine precision threshold. By contrast, for the first derivative, the complex step approximation decreases until it reaches the machine precision threshold for a step size of $10^{-8}$. For larger step sizes, both the finite difference and complex step approximations exhibit truncation error in the calculations of the first derivative. For step sizes smaller than $10^{-8}$, the finite difference calculations of the first derivative increase in error due to subtractive cancelation error.

Figure 2(b) assesses the calculations of the second derivative of Eq. 26. The hyper-dual number calculation again is limited only by the machine precision threshold as all of the higher order terms are zero by definition. The complex step approximation of the second derivative is unable to reach the machine precision threshold as the approximation is dominated by either truncation error or subtractive cancelation error.

\subsection{Parameterization Using Hyper-Dual Numbers}

For the present research, both second order and third order parameterizations are considered. One important question to address is what order parameterization is necessary to maximize the accuracy of the resulting PROM? For the beam application discussed in $\S 5$, terms such as the height of the beam enter into the system matrices as a cubic term. For the analytical solution, though, the height has a nonlinear relationship to the wave lengths of the natural frequencies (via the boundary conditions given in Appendix A). Similarly, the density of the beam $\rho$ enters into the analytical solution as the natural frequency $\omega \propto \sqrt{1 / \rho}$, but is linearly proportional to the terms in the system matrices. Thus, multiple orders of parameterizations are considered in order to study the ramifications of the choice of order of Taylor series expansion used.

A hyper-dual number implementation that produces exact third derivatives is created by including $\epsilon_{3}$ terms. This yields a Taylor series that truncates exactly at the third derivative term 


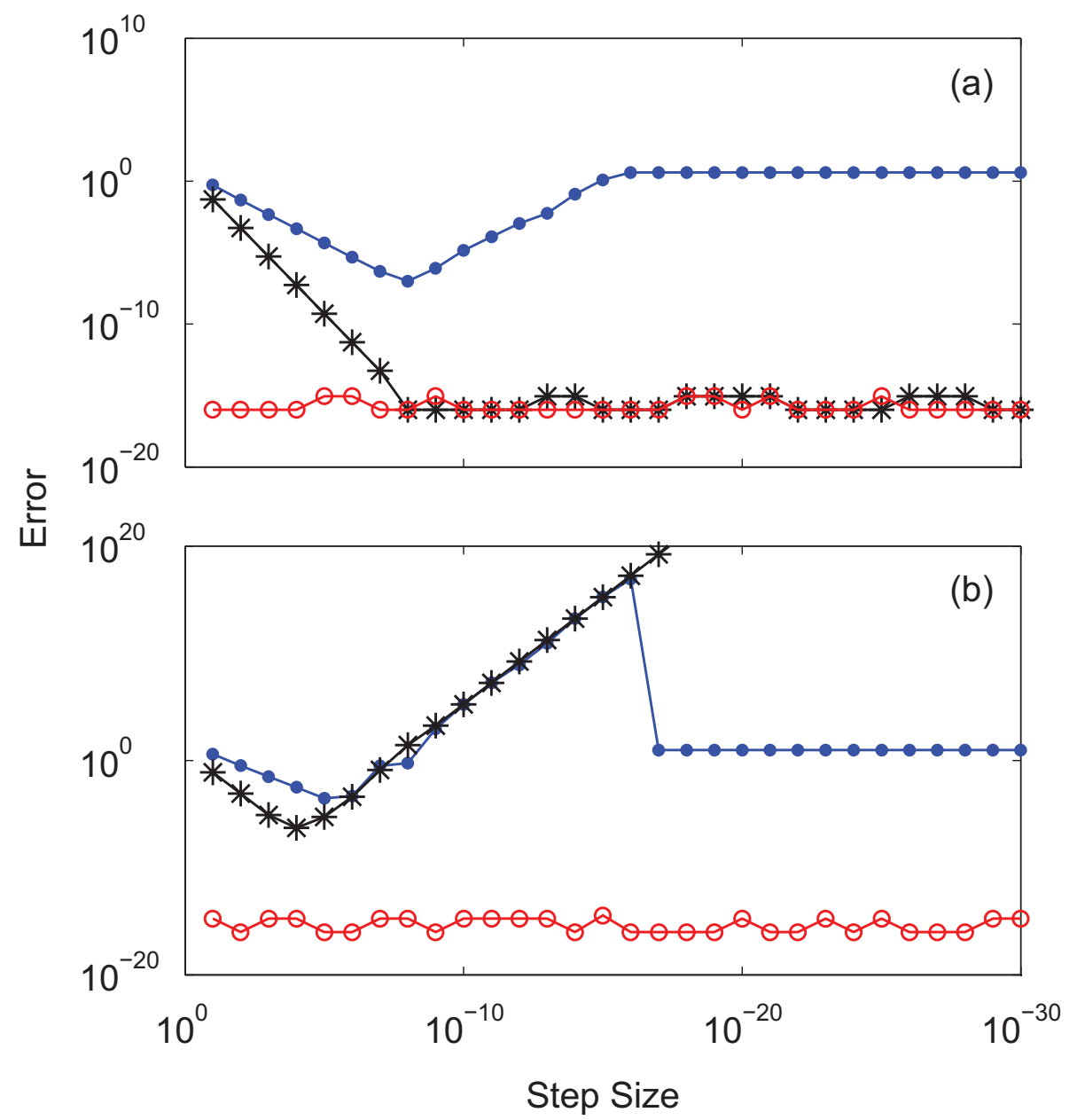

Figure 2: Absolute errors in the calculation of the (a) first and (b) second derivatives of Eq. 26 using a finite difference scheme (blue, - --), complex step approximation (black, $-*-$ ), and hyper-dual numbers (red, $-{ }^{-}$).

(with an arbitrary perturbation $h_{3}$ )

$$
\begin{aligned}
f\left(x+h_{1} \epsilon_{1}+h_{2} \epsilon_{2}+h_{3} \epsilon_{3}+0 \epsilon_{1} \epsilon_{2}+0 \epsilon_{1} \epsilon_{3}+0 \epsilon_{2} \epsilon_{3}+0 \epsilon_{1} \epsilon_{2} \epsilon_{3}\right) & \\
=f(x)+h_{1} f^{\prime}(x) & \epsilon_{1}+h_{2} f^{\prime}(x) \epsilon_{2}+h_{3} f^{\prime}(x) \epsilon_{3}+h_{1} h_{2} f^{\prime \prime}(x) \epsilon_{1} \epsilon_{2} \\
& +h_{1} h_{3} f^{\prime \prime}(x) \epsilon_{1} \epsilon_{3}+h_{2} h_{3} f^{\prime \prime}(x) \epsilon_{2} \epsilon_{3}+h_{1} h_{2} h_{3} f^{\prime \prime \prime}(x) \epsilon_{1} \epsilon_{2} \epsilon_{3} .
\end{aligned}
$$

The use of hyper-dual numbers requires overloading all of the functions in the analysis code to operate on hyper-dual numbers instead of on real numbers (that is, creating a new method for a function that operates on hyper-dual numbers instead of the originally intended data structure). However, there are often cases where routines are used for which the code is not available and 
therefore cannot be modified. In these situations it may still be possible to use hyper-dual numbers, if the effect of computing the derivatives can be achieved. A hyper-dual number function takes hyper-dual numbers as inputs and produces hyper-dual numbers as outputs. However, as long as the resulting hyper-dual number outputs are mathematically correct there is no need for the internal operations of the function to use hyper-dual numbers. This is discussed in detail in Appendix $\mathrm{B}$ for the calculation of derivatives of a matrix system solve and the derivatives of eigenvalues and eigenvectors, both of which are required for the Craig-Bampton Component Mode Synthesis method and which typically use routines for which the source code is unavailable.

\section{Analysis of a Stepped Beam}

The example application is a simply supported beam composed of three components - two narrow sections and a wide middle section, as shown in Fig. 3. The beam has elastic modulus $E$, density $\rho$, width $B$, cross-sectional height $H$, location of the center of the middle section $\ell$, and length of the middle section $W$, with nominal values given in Table 1. The material properties and geometry of the middle section are perturbed from their nominal values in the following analyses. This problem is chosen due to the existence of an exact, analytical solution given in Appendix A. The FE model of this system consists of approximately 80 beam elements to ensure converged results, and is found to match the analytical model in all subsequent analyses.

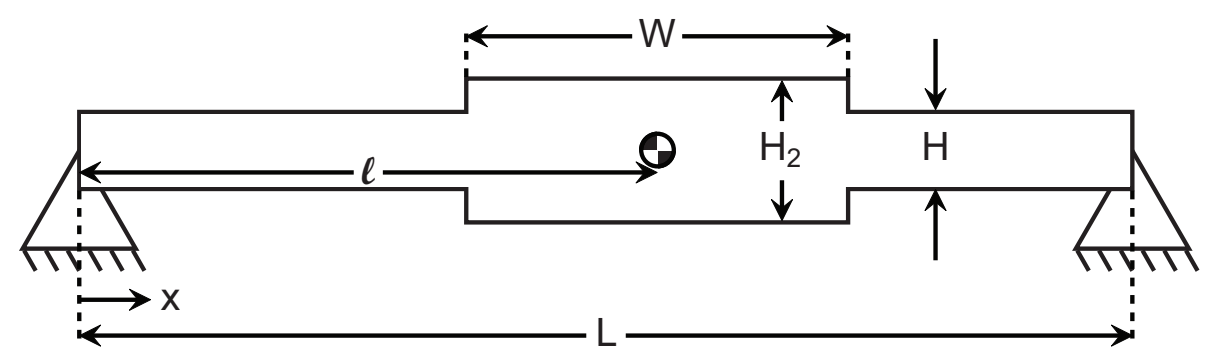

Figure 3: The geometry of the stepped beam used in the example application.

Parameterized models for this system are created using a Taylor series expansion (1). Second, third, and fourth order expansions are considered, with the derivatives computed using either hyper-dual numbers or finite difference approximations. Each of the levels of parameterization shown in Fig. 1 are presented in Figs. 4-8 for the length of the middle section, $W$, which is chosen as it exhibits a relatively large amount of error in the predictions of the natural frequencies by the 


\begin{tabular}{|lr|}
\hline Property & Value \\
\hline Density, $\rho$ & $2700 \mathrm{~kg} / \mathrm{m}^{3}$ \\
Density of the middle section, $\rho_{2}$ & $2700 \mathrm{~kg} / \mathrm{m}^{3}$ \\
Elastic modulus, $E$ & $68.9 \mathrm{GPa}$ \\
Elastic modulus of the middle section, $E_{2}$ & $68.9 \mathrm{GPa}$ \\
Cross-sectional width, $B$ & $20 \mathrm{~mm}$ \\
Cross-sectional width of middle section, $B_{2}$ & $30 \mathrm{~mm}$ \\
Cross-sectional height, $H$ & $20 \mathrm{~mm}$ \\
Cross-sectional height of middle section, $H_{2}$ & $30 \mathrm{~mm}$ \\
Location of middle section's center, $\ell$ & $45 \mathrm{~cm}$ \\
Length of the middle section, $W$ & $30 \mathrm{~cm}$ \\
Length of the beam, $L$ & $1 \mathrm{~m}$ \\
\hline
\end{tabular}

Table 1: Material and geometric properties for the beam.

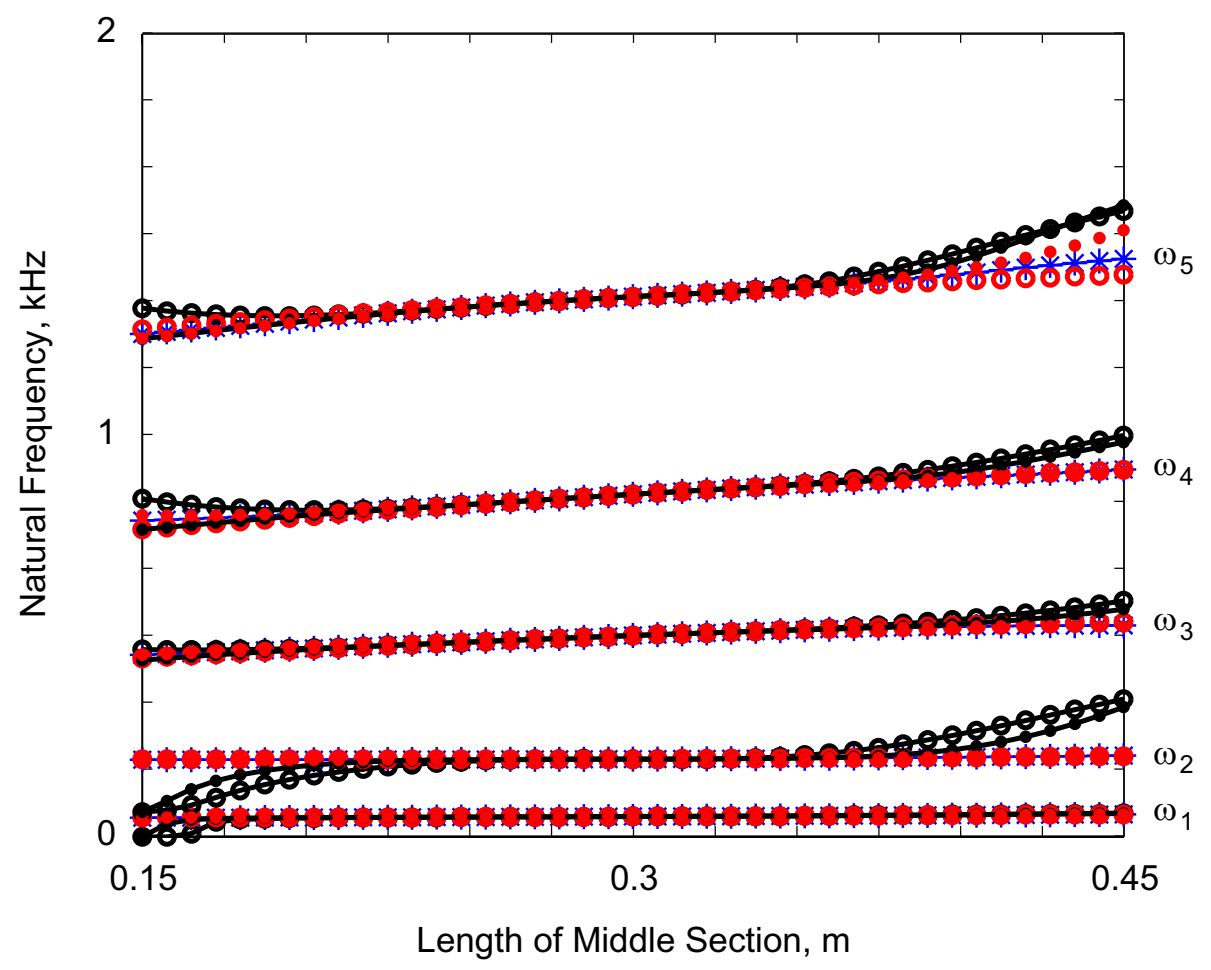

Figure 4: Comparison of the FDPs for second-order Taylor series expansions of the CMS matrices (black, -o-), second-order expansions of the eigenvalues (red, ०), fourth-order expansions of the CMS matrices (black, - $\bullet-$ ), and fourth-order expansions of the eigenvalues (red, •) with the exact solution (blue, $*$ ) as functions of the length of the middle section, $W$. The first $\omega_{1}$ through fifth $\omega_{5}$ natural frequencies are indicated on the right side of the figure.

parameterizations compared to the other variables of interest. Note that while natural frequencies scale linearly with $W$ for a uniform beam, because the system of Fig. 3 is composed of three 
contiguous sections and only the properties of the middle section are being varied, there is a nonlinear relationship between the geometric property $W$ and the natural frequencies. At the scale presented in the subsequent figures, this is difficult to discern, but closer inspection shows that there is a nonlinear effect that strongly influences the local gradients. Thus, this parameterization should present a challenge for the hyper-dual number approach, which is based on local gradients, more so than for the finite difference approach, which is based on more global calculations.

To begin with, finite differences are used to compute derivatives and a comparison is made between second and fourth order Taylor series expansions. Next, derivatives are computed using hyper-dual numbers, and a comparision is made between second and third order Taylor series expansions for the FE branch of Fig. 1. A comparison is then made between CMS using all the modes versus a truncated number of modes for both second and third order hyper-dual parameterizations. This level of comparison is made to have a thorough understanding of the method and to assess the accuracy of the CMS formulation. Then, comparisons are made for the other variables of interest for both hyper-dual parameterizations and finite difference parameterizations.

Figure 4 presents the finite difference parameterizations (FDP) of both the CMS matrices and the eigenvalues for second and fourth order Taylor series expansions. The fourth order Taylor series expansions show better agreement with the exact, analytical solution for the eigenvalues. As well, the eigenvalue parameterization consistently is more accurate in predicting the actual eigenvalues than the CMS parameterization; however, all methods show reasonably good agreement with the analytical solution.

The accuracy of the FDPs are dependent upon the step size used for the Taylor series approximations ( $h$ in $\S 3$ ), as shown in Fig. 5 for the parameterization of the length of the middle section, $W$. For the results shown in Fig. 4, the finite difference step size is defined to be $\pm 10 \%$ (for both the second and fourth order FDPs) and $\pm 20 \%$ (for the two additional meshes needed for the fourth order FDPs) of the nominal values (i.e. $h=0.03 \mathrm{~m}$ ). An important consideration for the FDPs is the range of predictability, i.e. the range over which the error of the parameterization is below some error threshold. For the FDPs, the range of predictability is dependent on the finite difference step size used to construct the parameterizations. Figure 5 shows the range of predictability for multiple error levels for FDPs of the FE matrices, CMS matrices, and eigenvalues. To interpret this figure, the diagonal blue line indicates when the range of predictability of a method is equal to 


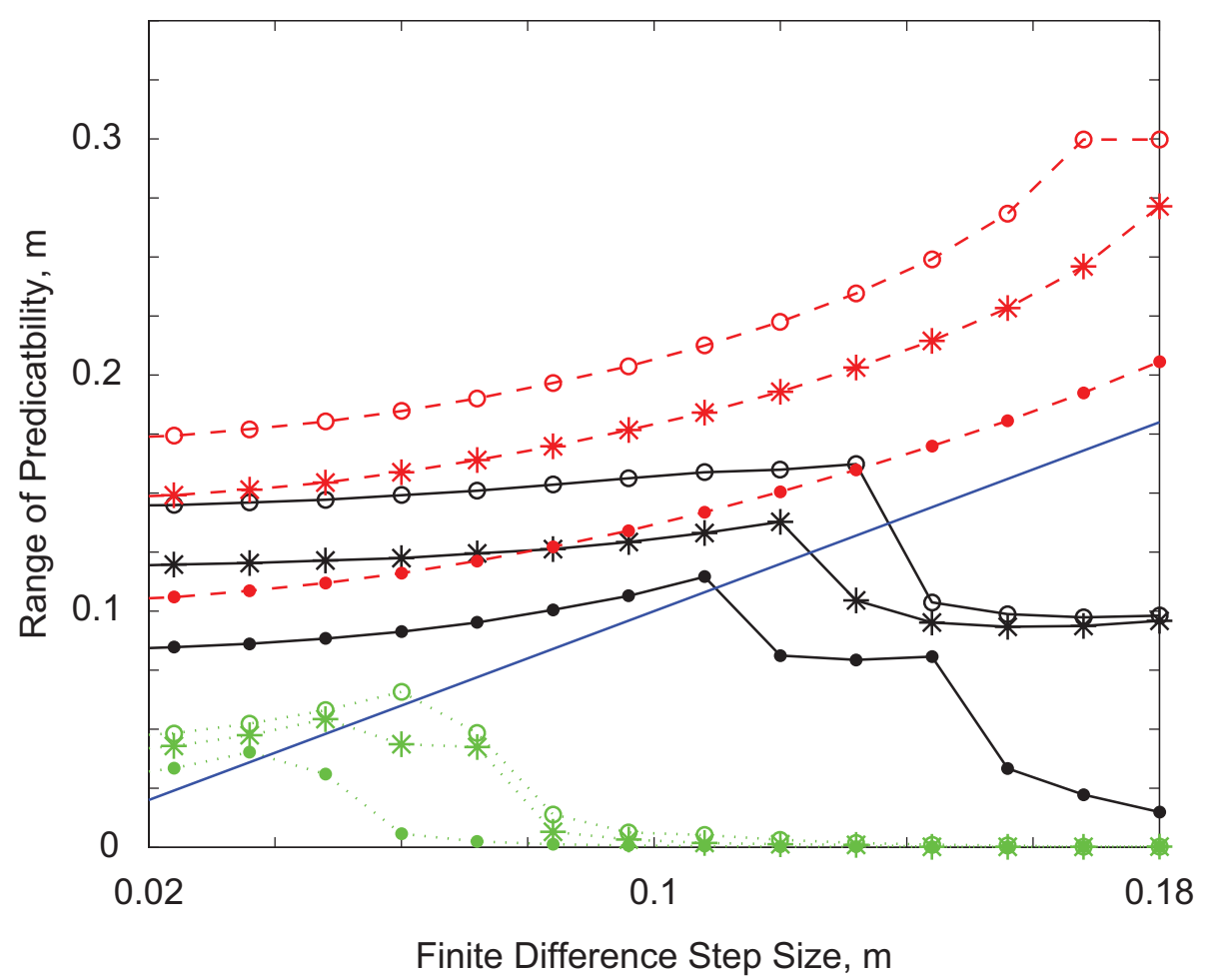

Figure 5: Range of predictability of the parameterization of the length of the middle section, $W$, as a function of the finite difference step size for the FDP of the FE matrices (green, ...), the CMS matrices (black, - ), and the eigenvalues (red, - -). The range of predictability is defined as the range over which a parameterization has less than a specified amount of error. Shown are curves denoting $1 \%$ error $(\bullet), 5 \%$ error $(*)$, and $10 \%$ error (o). The solid, diagonal line indicates when the range of predictability is equal to the extent of the meshes used for the Taylor series approximations.

the extent of the models used for the Taylor series approximations. Values above this line indicate that the model is predictive outside of the range that it is calculated over, while values below this line indicate that the model is predictive over a range smaller than it is calculated over, a non-ideal situation as this means that the accuracy of the model is questionable over the regime that it should be accurate. Similar to the results of Fig. 4, the FDP of the eigenvalues has the greatest range of predictability for $W$ compared to the other FDPs for a given error percentage. At some point for all parameters investigated, the range of predictability becomes less than the extent of the finite difference step size. For example, this occurs for the FDP of the FE matrices at a finite difference step size of $h=0.04 \mathrm{~m}$ with an error of $1 \%$, and for the FDP of the CMS matrices at $h=0.11 \mathrm{~m}$ with an error of $1 \%$. Thus, in using the FDP, it is important to assess the range of predictability for the specific application. 


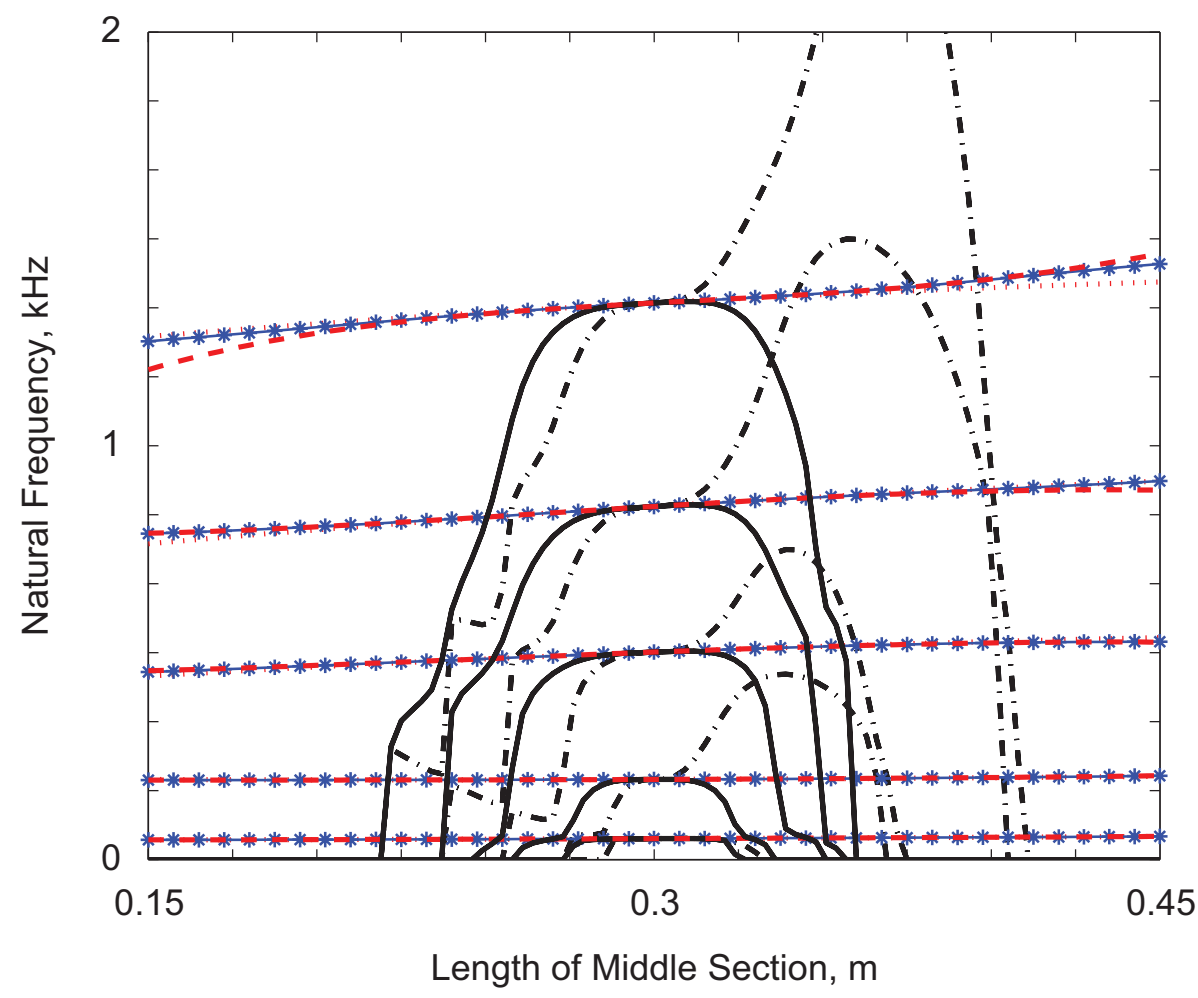

Figure 6: Comparison of the HDPs of the FE models comparing the second-order parameterization of the system matrices (black, - - -), the second-order parameterization of the eigenvalues (red, $\cdots$ ), the third-order parameterization of the system matrices (black, - ), and the third-order parameterization of the eigenvalues (red, --) with the exact solution (blue, $-*-$ ) as functions of the length of the middle section, $W$.

The hyper-dual number parameterization (HDP) of the FE model (without any model reduction) is shown in Fig. 6. The predictions made by parameterizing the FE matrices exhibits a significant amount of error away from the nominal value, with the third order HDP showing less error than the second order HDP. This result is expected as it is non-trivial to account for the redistribution of nodes in the parameterization of the FE matrices. For comparison, the parameterization of the eigenvalues predicted by the high fidelity FE model are shown, and good agreement is observed for both the second order and third order HDPs.

Figure 7 shows the second order HDPs of the CMS matrices and of the eigenvalues. These parameterizations include both a parameterization of the CMS matrices that preserves the number of DOFs of the entire model (referred to as full CMS hereafter), and a reduced model that contains 36 DOFs (referred to as reduced CMS hereafter). For both the full and reduced CMS, the eigenvalue parameterizations show good agreement with the exact solution. The parameterizations of the 


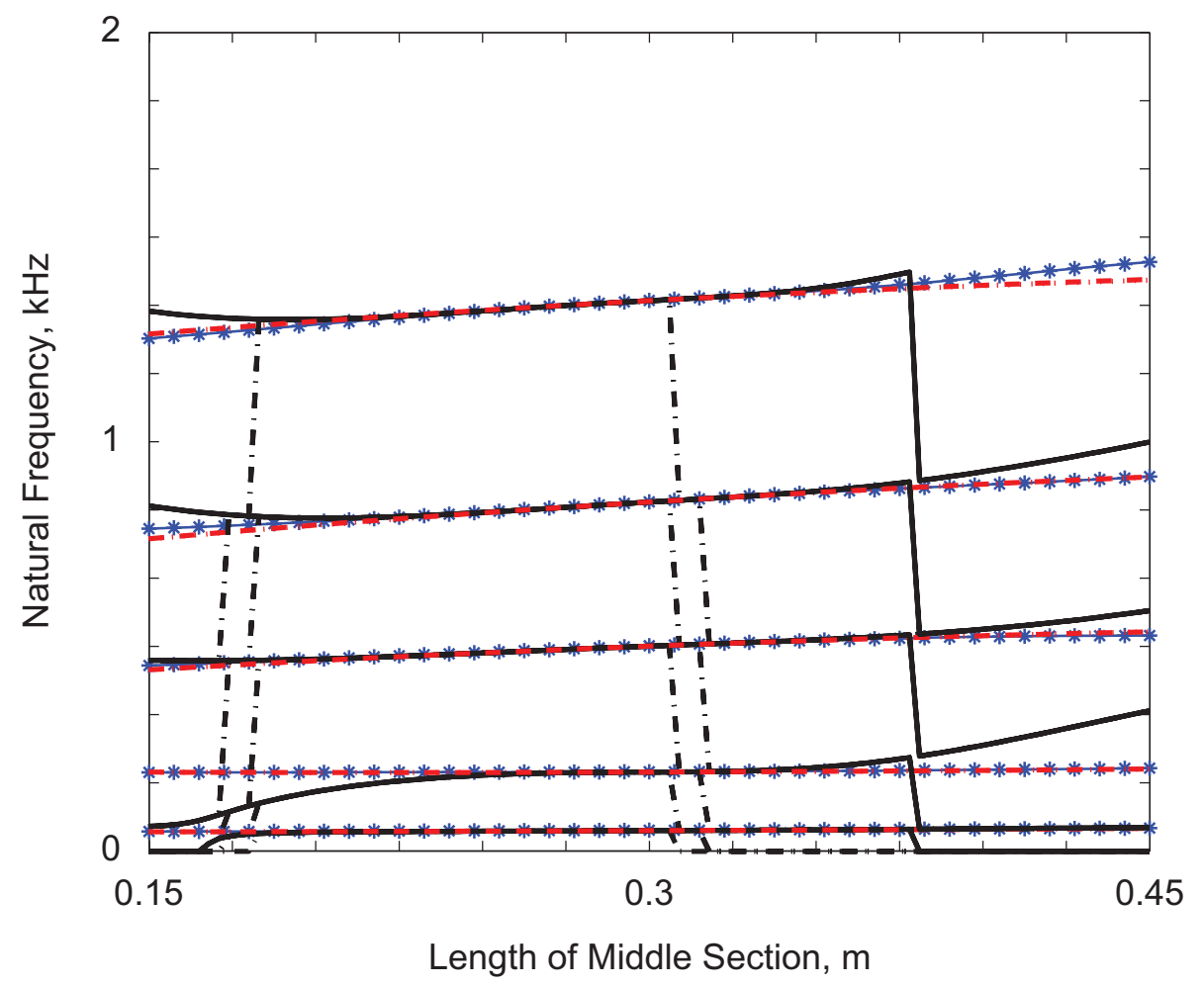

Figure 7: Comparison of the second-order HDPs for the full CMS matrices (black, - - -), the full CMS eigenvalues (red, $\cdots$ ), the reduced CMS matrices (black, - ), and the reduced CMS eigenvalues (red, --) with the exact solution (blue, $-*-$ ) as functions of the length of the middle section, $W$.

CMS matrices, however, show that the full CMS has a smaller range of predictability than the reduced CMS, and that the parameterization of the reduced CMS exhibits mode veering as the first eigenvalue goes to zero for $W>0.38 \mathrm{~m}$. It is hypothesized that the full CMS parameterizations are less accurate due to the parameterizations not guaranteeing that the positive definite quality of the matrices is preserved for values that are too large for the parameterization. Future work on this method should focus on developing a mathematically rigorous approach to ensure that the necessary matrix properties (such as positive definiteness) are preserved.

Similar results are observed for the third order HDPs in Fig. 8. The parameterizations of the eigenvalues are the most accurate for predicting the eigenvalues, and the parameterizations of the reduced CMS are more accurate than of the full CMS. In both Figs. 7 and Figs. 8, the parameterizations of the component matrices are indistinguishable from the parameterizations of the system matrices (the multiple levels of CMS parameterizations in Fig. 1), which implies that parameterizing then assembling is equally as accurate as assembling then parameterizing. 


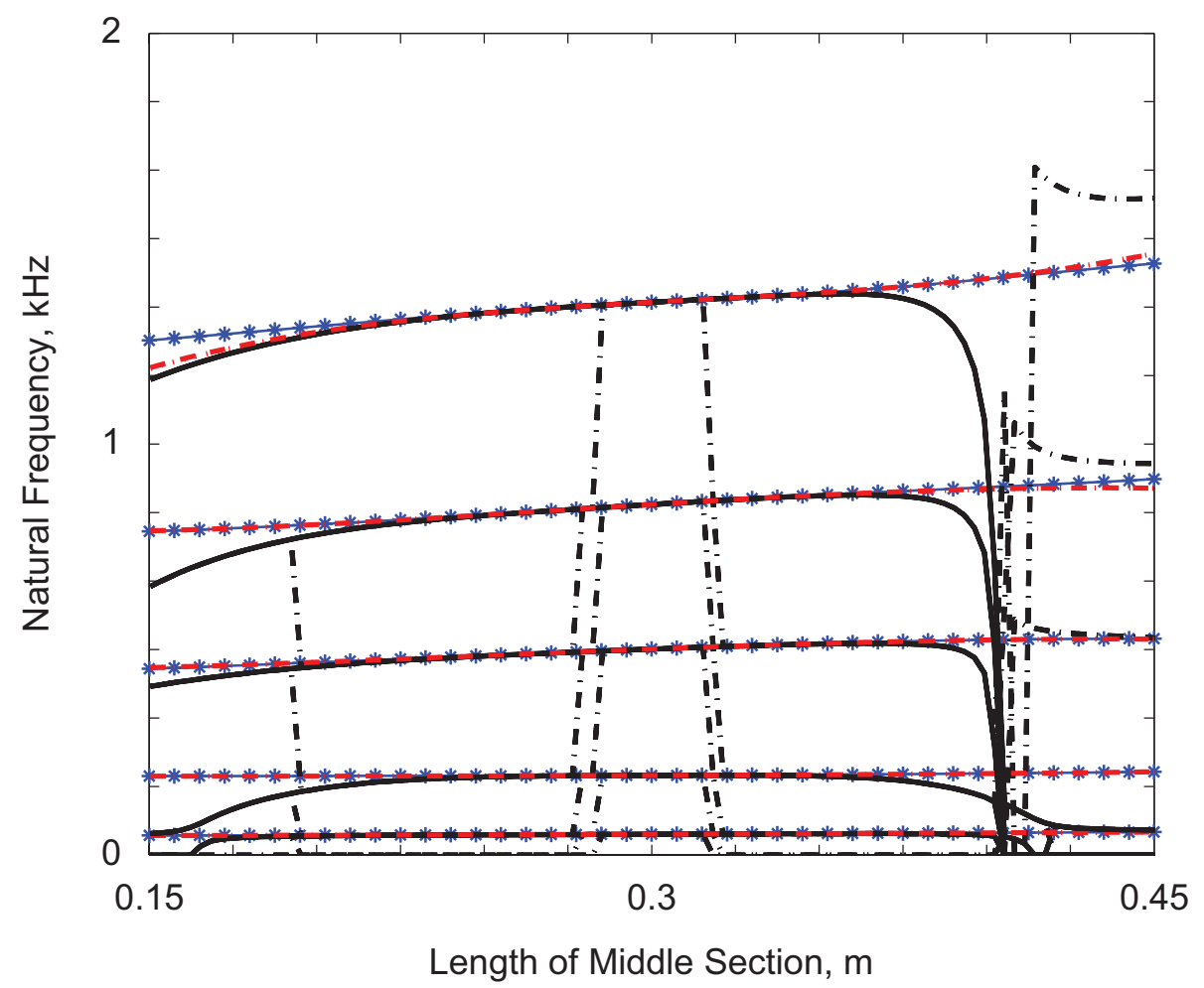

Figure 8: Comparison of the third-order HDPs for the full CMS matrices (black, - - -), the full CMS eigenvalues (red, $\cdots$ ), the reduced CMS matrices (black, - ), and the reduced CMS eigenvalues (red, --) with the exact solution (blue, $-*-$ ) as functions of the length of the middle section, $W$.

Several preliminary conclusions for univariate PROMs are able to be made from the results of Figs. 4-8:

- Parameterizations of reduced CMS matrices are more accurate than parameterizations of CMS matrices that preserve the total number of DOFs.

- Parameterizations of the quantities of interest (eigenvalues in this example) are more accurate than parameterizing other levels of the model.

- Parameterizations of component matrices then assembling the system matrices is equally as accurate as assembling the system matrices then parameterizing.

- Parameterizations using finite difference Taylor series are more accurate than hyper-dual numbers in the manner that they are implemented in this research (seen from comparing Fig. 4 to Figs. 6-8) due to the local nature of hyper-dual parameterizations. 
With regards to the last preliminary conclusion, this is an expected result. The FDPs are based on multiple FE models, which allows for the parameterization to represent more accurately how the system changes with a parameter, while the HDPs are based upon a single mesh. For systems with only a few variables of interest for parameterization, the finite difference approach is viable; however, as the number of parameters of interest is increased, the number of FE meshes needed to derive the Taylor series expansions geometrically increases from five meshes for a fourth order Taylor series expansion of a single variable to 301 meshes for a fourth order Taylor series expansion of five variables.

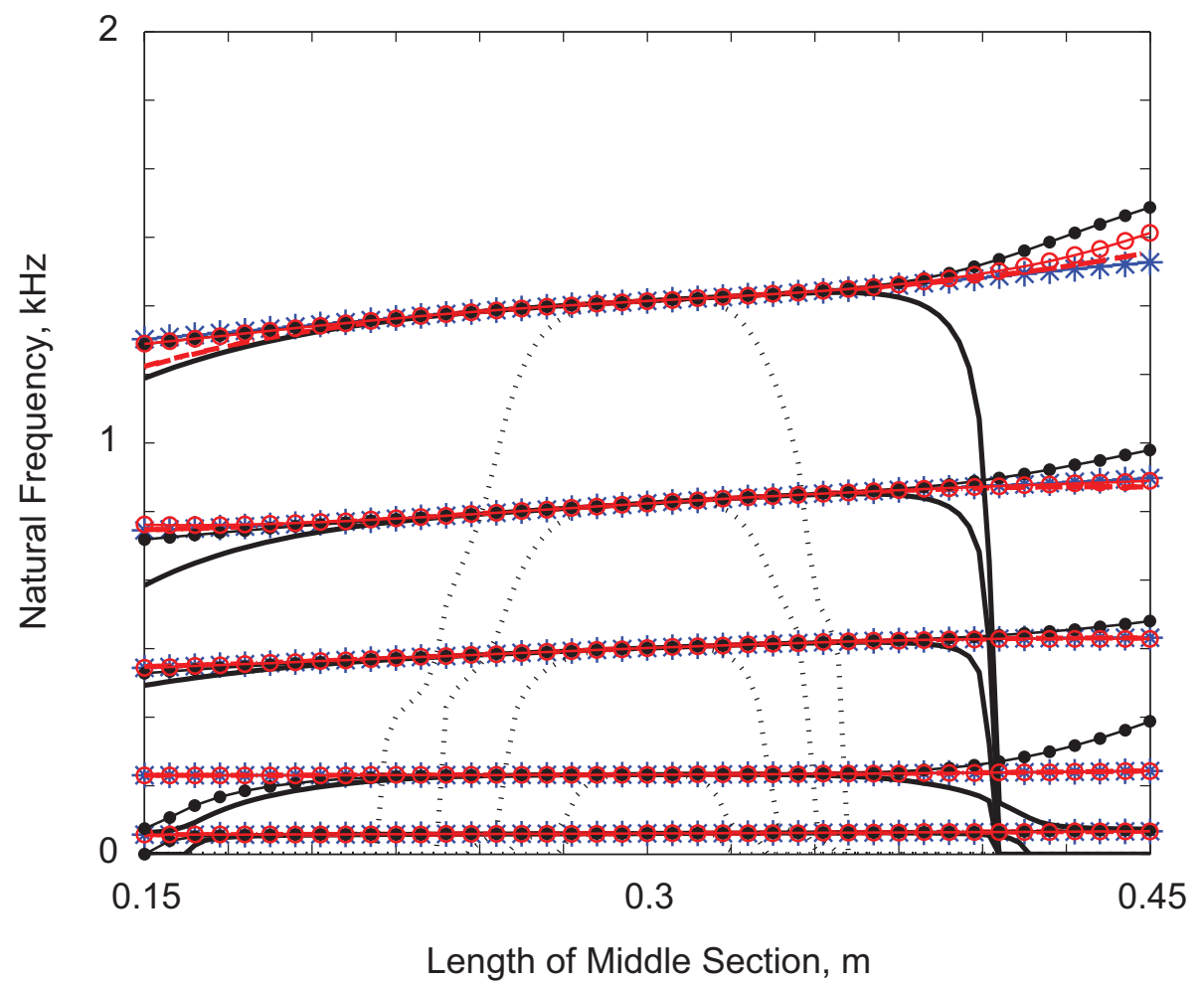

Figure 9: Comparison of the different parameterization schemes as functions of the length of the middle section, $W$, to the analytical solution (blue, $-*-$ ) for the fourth order FDP of the CMS matrices (black, $-\bullet-$ ), the fourth order FDP of the eigenvalues (red, - - -), the 3rd order HDP of the FE matrices (black, $\cdots$ ), the 3rd order HDP of the eigenvalues calculated directly from the FE matrices (red, - - -), the 3rd order HDP of the CMS matrices (black, - ), and the 3rd order HDP of the eigenvalues calculated from the CMS matrices (red, --).

Several of the different parameterization schemes are compared to each other in Fig. 9 as functions of the length of the middle section, $W$. For most parameters, the eigenvalue HDPs show no qualitative difference in their predictions. For the first through fourth modes, the eigenvalue HDPs appear qualitatively the same as the eigenvalue FDP; for the fifth mode, and higher (not 
shown), the HDPs of the eigenvalues show less error for large parameter changes than the FDP of the eigenvalues. For all modes, the FDP of the CMS matrices is more accurate than the HDP of the CMS matrices, and both of those methods are more accurate than the HDP of the FE matrices. Even though the HDP of the CMS matrices is constructed from a single mesh, it exhibits qualitative agreement with the analytical solution for parameter changes of up to $\pm 20 \%$ of the nominal value. By contrast, the third order HDP of the FE matrices is only valid over a small range as the matrices become ill conditioned for moderate sized perturbations (i.e. the natural frequencies tending towards zero).

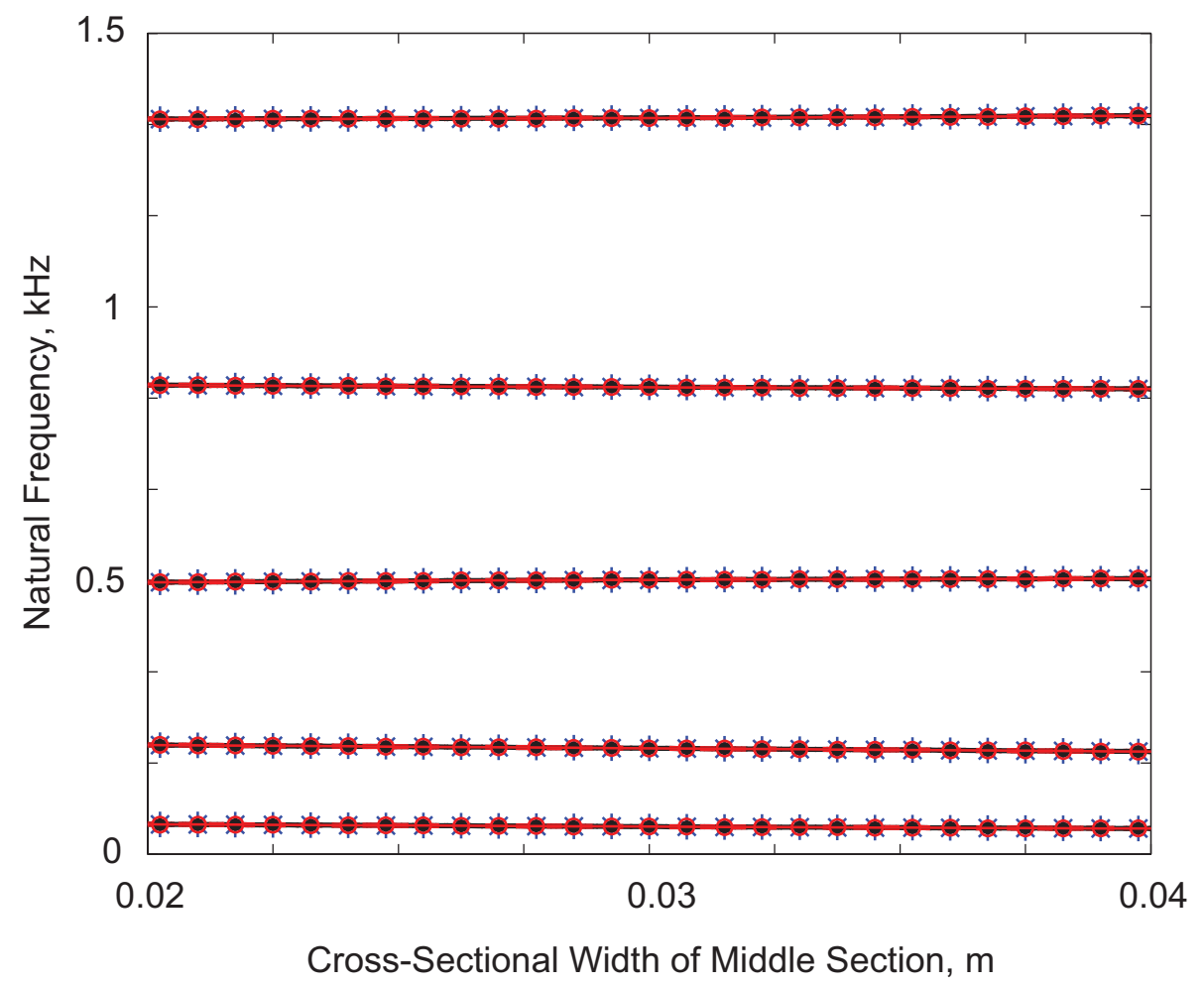

Figure 10: Comparison of the different parameterization schemes as functions of $B_{2}$ to the analytical solution (blue, $-*-)$ for the fourth order FDP of the CMS matrices (black, $-\bullet$ ), the fourth order FDP of the eigenvalues (red, -०-), the 3rd order HDP of the FE matrices (black, $\cdots$ ), the 3rd order HDP of the eigenvalues calculated directly from the FE matrices (red, - - -), the 3rd order HDP of the CMS matrices (black, - ), and the 3rd order HDP of the eigenvalues calculated from the CMS matrices (red, --).

In contrast to the parameterizations of the length of the middle section, $W$, most parameters show high levels of agreement for $\pm 50 \%$ variations of the nominal values, as seen in Figs. 10-13 for $B_{2}, H_{2}, E_{2}$, and $\rho_{2}$ respectively. For each of these four parameters, no rearrangements of the nodes are required for the FE mesh as one-dimensional beam elements are used. This is in contrast to the 
change in elements inherent in changing $W$ (Fig. 9) or $\ell$ (Fig. 14). For the parameterization of $B_{2}$ (Fig. 10), the full CMS HDP (both for second order and third order HDPs, not shown for brevity) exhibits similar behavior as seen in Fig. 7, in which the first natural frequency goes to zero, and the second and higher natural frequencies drop to a lower mode. Other than the full CMS HDPs, all results show excellent qualitative agreement with the analytical solution. These, and other results, are quantified in Tables 2 and 3.

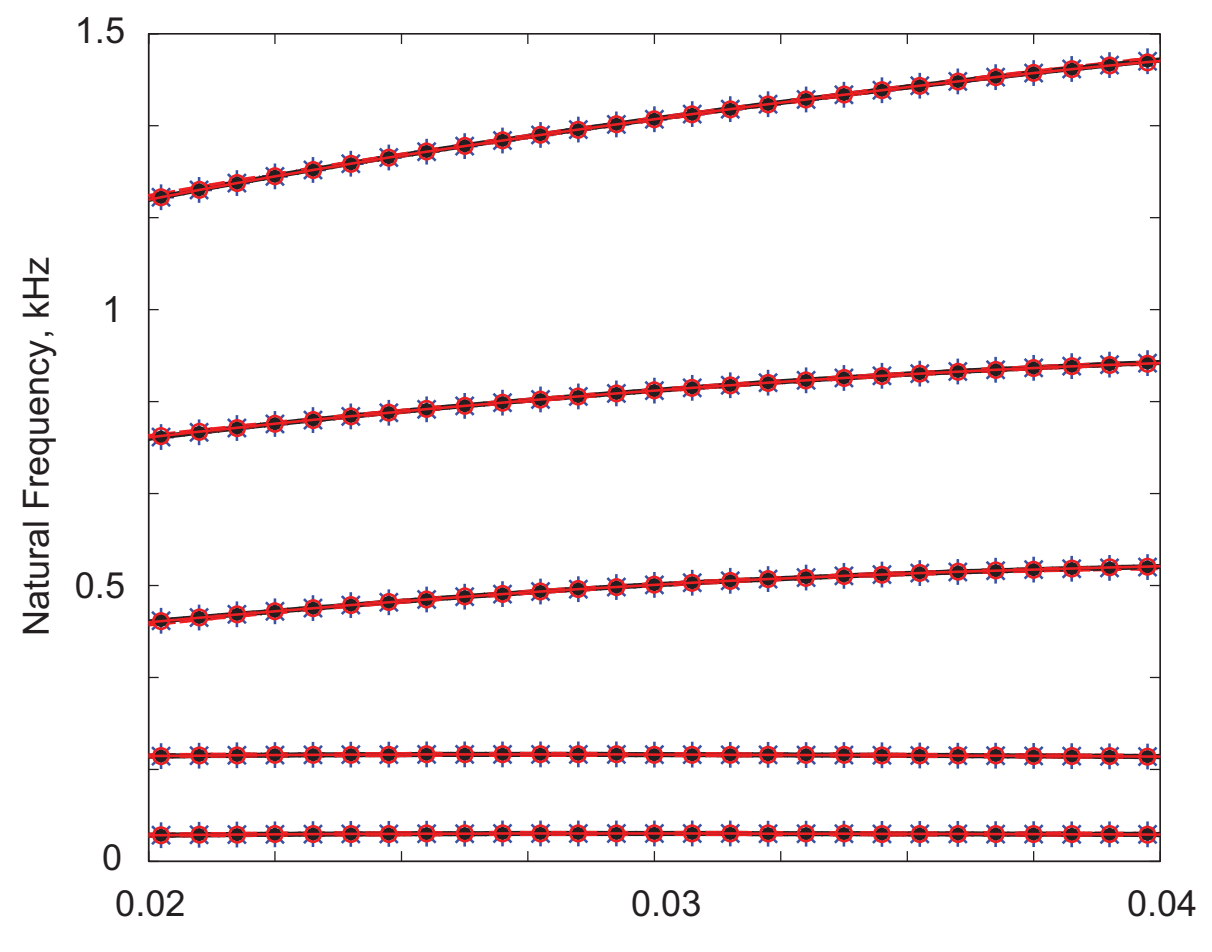

Cross-Sectional Height of Middle Section, m

Figure 11: Comparison of the different parameterization schemes as functions of $H_{2}$ to the analytical solution (blue, $-*-$ ) for the fourth order FDP of the CMS matrices (black, - - ), the fourth order FDP of the eigenvalues (red, -०-), the 3rd order HDP of the FE matrices (black, $\cdots$ ), the 3rd order HDP of the eigenvalues calculated directly from the FE matrices (red, - - -), the 3rd order HDP of the CMS matrices (black, - ), and the 3rd order HDP of the eigenvalues calculated from the CMS matrices (red, --).

Similar to $B_{2}$, the parameterizations of $H_{2}$ (Fig. 11) show excellent agreement for all HDPs and FDPs. In assessing the quantitative comparisons presented later in Tables 2 and 3, the HDPs of the full CMS and the FE HDPs, for both the second order and third order HDPs (though not seen at the scale in Fig. 11), small errors are apparent. Further, the eigenvalue HDPs are found to be less accurate than the CMS HDPs (though this trend does not persist for the FDP). In the parameterizations of $E_{2}$ (Fig. 12), a similar trend is observed as for $H_{2}$ : the eigenvalue HDPs, while 
exhibiting high qualitative agreement, are not as accurate as the CMS HDPs. These results are further explored later with the discussion of Tables 2 and 3.

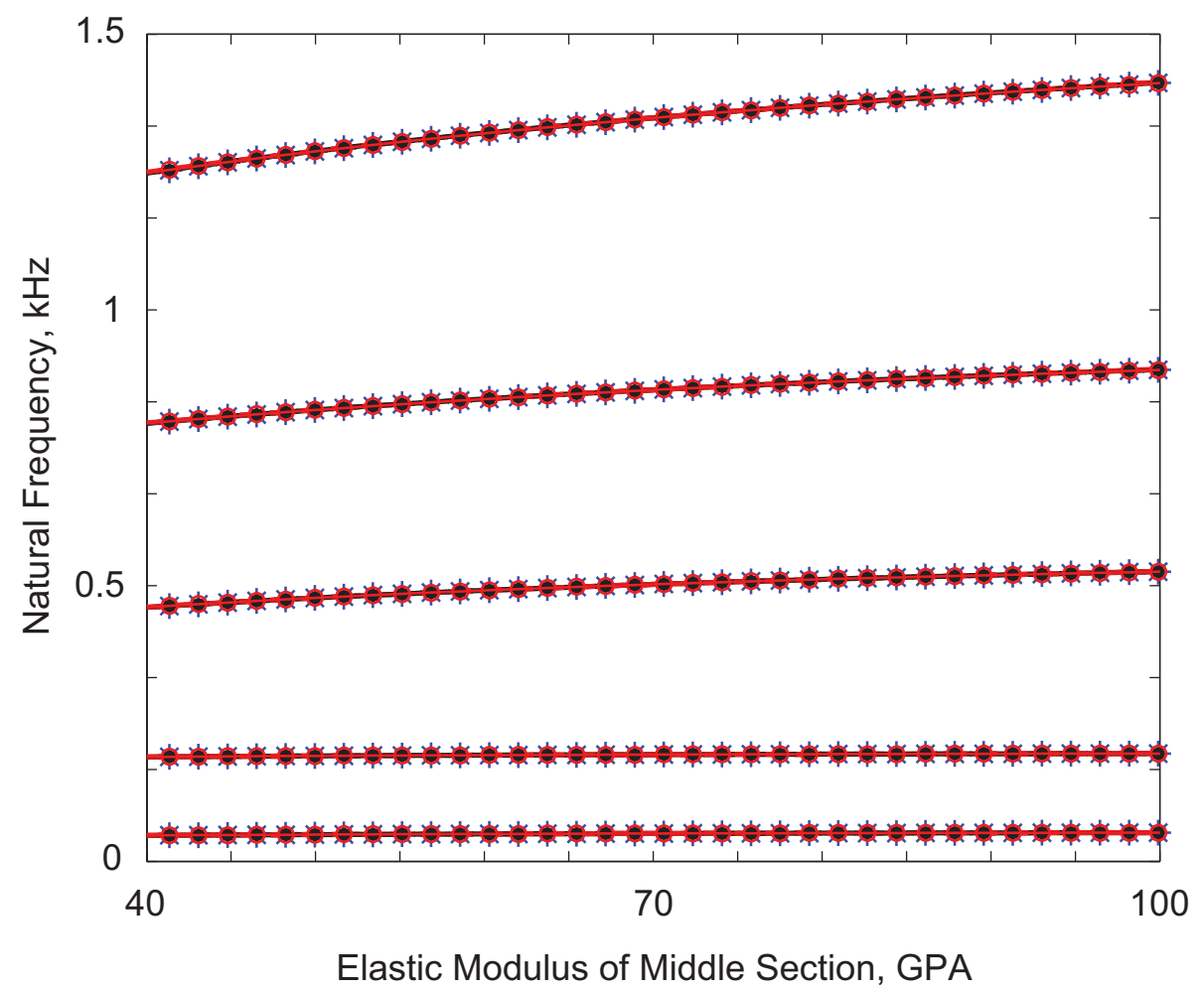

Figure 12: Comparison of the different parameterization schemes as functions of $E_{2}$ to the analytical solution (blue, $-*-)$ for the fourth order FDP of the CMS matrices (black, - $\bullet-$ ), the fourth order FDP of the eigenvalues (red, -०-), the 3rd order HDP of the FE matrices (black, $\cdots$ ), the 3rd order HDP of the eigenvalues calculated directly from the FE matrices (red, - . -), the 3rd order HDP of the CMS matrices (black, - ), and the 3rd order HDP of the eigenvalues calculated from the CMS matrices (red, --).

The parameterization of $\rho_{2}$, presented in Fig. 13, shows identical trends as to the parameterization of $B_{2}$. The eigenvalue HDPs show high qualitative agreement, but quantitatively exhibit more error than the CMS HDPs. The full CMS HDPs (not shown) exhibit behavior similar to Fig. 7 in which the first natural frequency goes to zero, and the higher natural frequencies jump down.

In contrast to the parameterizations of $B_{2}, H_{2}, E_{2}$, and $\rho_{2}$, the parameterization of $\ell$ requires the change of nodal positions in the high fidelity FE model, similar to the length of the middle section, $W$; consequently, less qualitative agreement is observed between the analytical solution and the predictions of the HDPs and FDPs, as shown in Fig. 14. Similar trends as to $W$ persist for $\ell$ : the HDP of the FE matrices exhibits the worst agreement of all models shown, followed by the HDP 


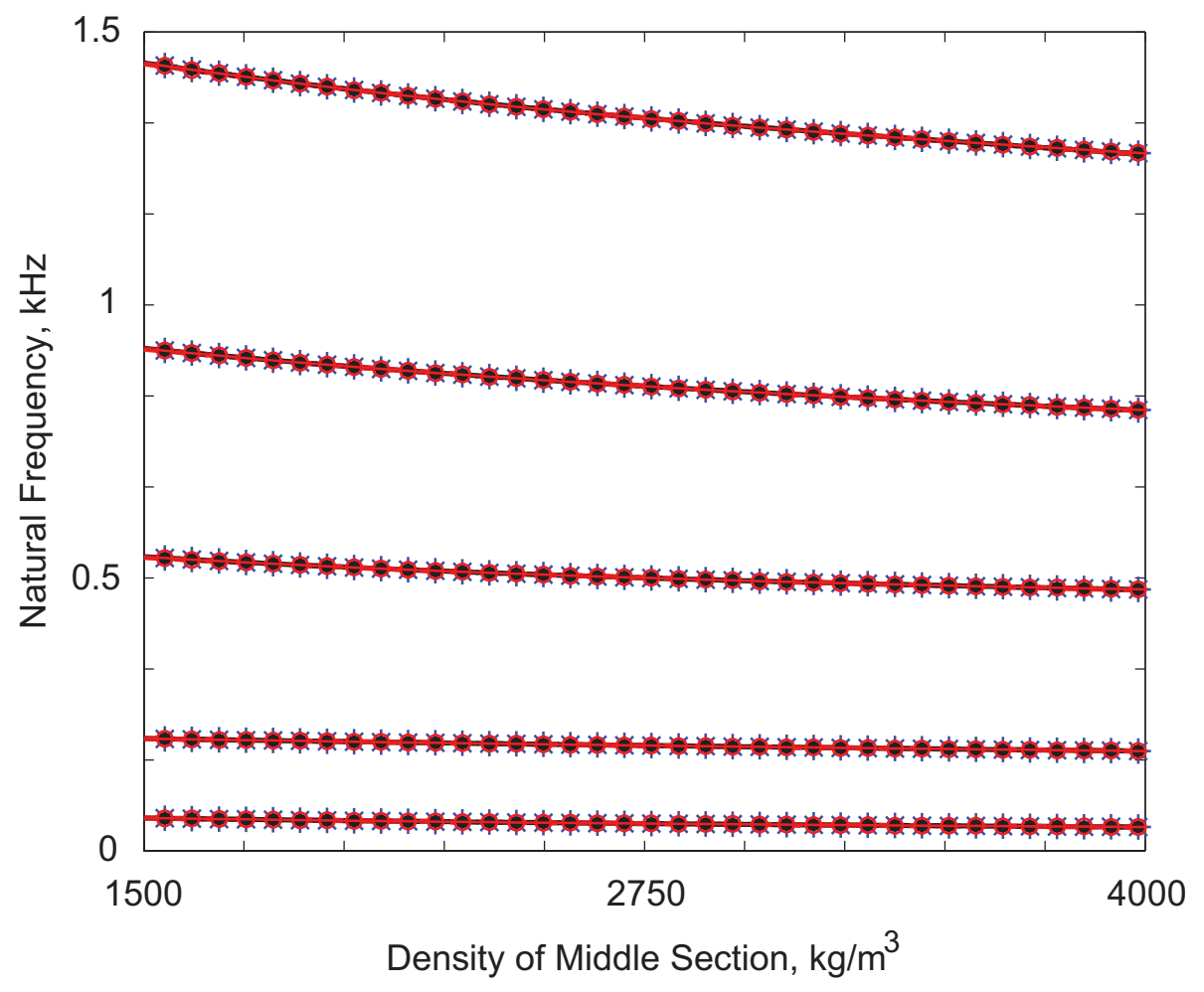

Figure 13: Comparison of the different parameterization schemes as functions of $\rho_{2}$ to the analytical solution (blue, $-*-$ ) for the fourth order FDP of the CMS matrices (black, - $\bullet-$ ), the fourth order FDP of the eigenvalues (red, -०-), the 3rd order HDP of the FE matrices (black, $\cdots$ ), the 3rd order HDP of the eigenvalues calculated directly from the FE matrices (red, - - -), the 3rd order HDP of the CMS matrices (black, - ), and the 3rd order HDP of the eigenvalues calculated from the CMS matrices (red, --).

of the CMS matrices and the FDP of the CMS matrices. Each of the eigenvalue parameterizations shows similar levels of agreement, though distinct differences are observed in the different methods used to derive the eigenvalue parameterizations.

Tables 2 (for $B_{2}, H_{2}$, and $E_{2}$ ) and 3 (for $\rho_{2}, \ell$, and $W$ ) quantify the range over which each parameterization can predict each parameter of interest with less than $1 \%$ error. The results in the tables are bounded by $-99 \%$ (as $-100 \%$ corresponds to a parameter value of zero) and $1000 \%$ (chosen as an arbitrarily large number). Several trends are able to be discerned from the data: for the FDPs, the CMS parameterizations are more accurate than the eigenvalue parameterizations for $B_{2}, H_{2}, E_{2}$, and, to some extent, $\rho_{2}$. This is in contrast with the observations of the HDP eigenvalue and CMS parameterizations. A second trend is that the fourth order FDPs are more accurate than the second order FDPs; however, one exception to this generalization is the prediction of $W$ using eigenvalue parameterizations. A similar trend is observable as the third order HDPs generally are 


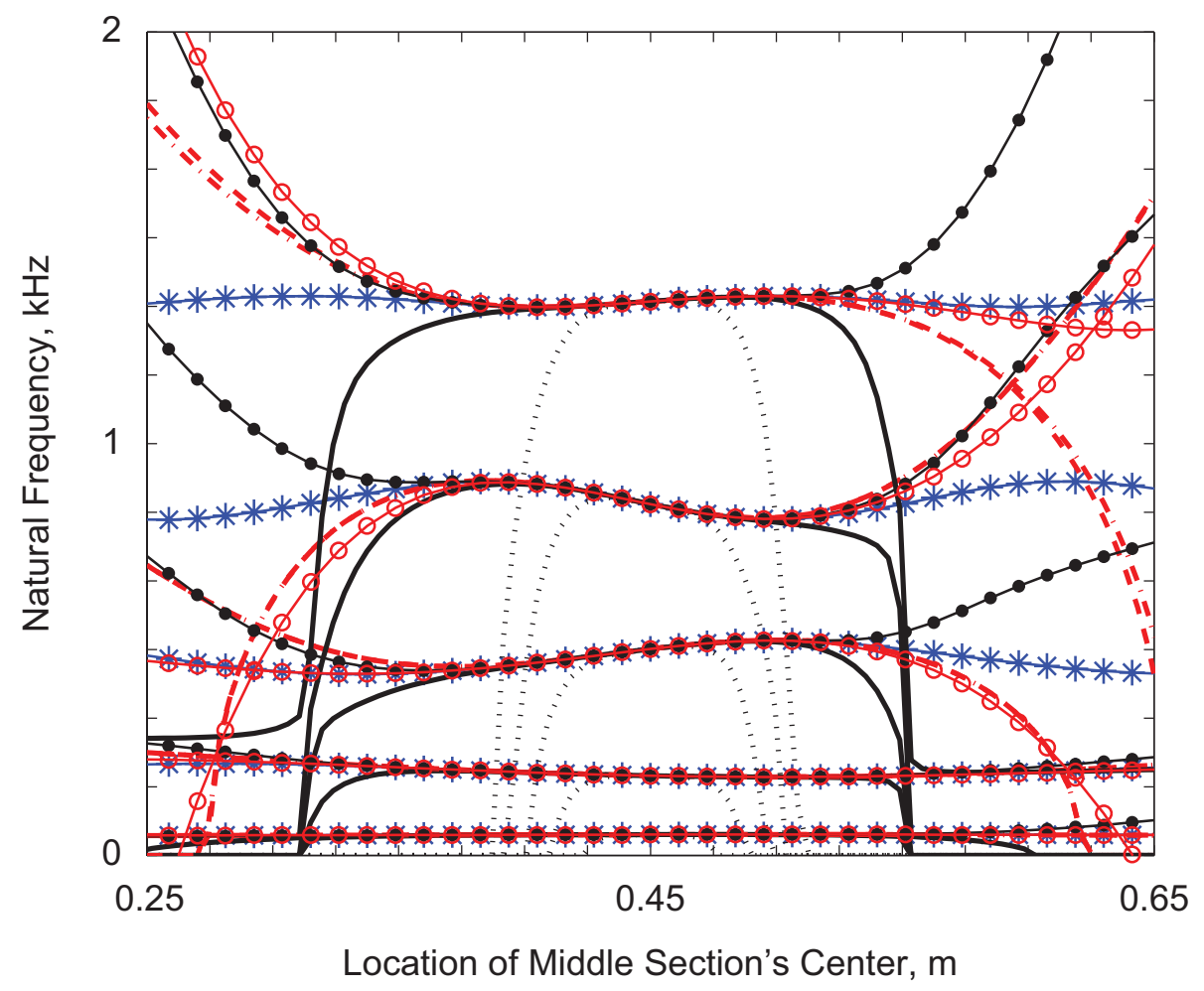

Figure 14: Comparison of the different parameterization schemes as functions of $\ell$ to the analytical solution (blue, $-*-$ ) for the fourth order FDP of the CMS matrices (black, - $\bullet-$ ), the fourth order FDP of the eigenvalues (red, -o-), the 3rd order HDP of the FE matrices (black, $\cdots$ ), the 3rd order HDP of the eigenvalues calculated directly from the FE matrices (red, - - -), the 3rd order HDP of the CMS matrices (black, - ), and the 3rd order HDP of the eigenvalues calculated from the CMS matrices (red, --).

more accurate than the second order HDPs, with a few exceptions (such as using the full CMS parameterizations to predict $W$ ).

Another observation is that $E_{2}$ is able to be predicted extremely accurately by all methods except for the eigenvalue parameterizations. This is due to both the FE matrices and CMS matrices being linearly dependent on $E_{2}$, while the eigenvalues have a nonlinear dependence on $E_{2}$. Similarly, the FE HDPs are both able to predict the natural frequencies for extremely large ranges of $\rho_{2}$, which makes sense as the mass matrices are linearly dependent upon $\rho_{2}$ while the CMS matrices and eigenvalues are nonlinearly dependent upon $\rho_{2}$.

In inspecting the range of validity for the HDPs, several trends are evident. First, the FE parameterizations are quite accurate for parameters that do not change the nodal locations (since one-dimensional beam elements are used, the only parameters that change the nodal locations are the length of the middle section, $W$, and the location of the middle section, $\ell$ ). Unexpectedly, 


\begin{tabular}{|lrrr|}
\hline Method & $\begin{array}{r}\text { Middle Section's } \\
\text { Width, } B_{2}\end{array}$ & $\begin{array}{r}\text { Middle Section's } \\
\text { Height, } H_{2}\end{array}$ & $\begin{array}{r}\text { Middle Section's } \\
\text { Modulus, } E_{2}\end{array}$ \\
\hline FDP, $2^{\text {nd }}$ Order CMS & {$[-85 \%, 327 \%]$} & {$[-32 \%,>1000 \%]$} & {$[<-99 \%,>1000 \%]$} \\
FDP, $2^{\text {nd }}$ Order Eigenvalues & {$[-95 \%, 152 \%]$} & {$[-38 \%, 70 \%]$} & {$[-50 \%, 74 \%]$} \\
\hline FDP, $4^{\text {th }}$ Order CMS & {$[-91 \%, 228 \%]$} & {$[-69 \%, 844 \%]$} & {$[-99 \%,>1000 \%]$} \\
FDP, $4^{\text {th }}$ Order Eigenvalues & {$[-93 \%, 231 \%]$} & {$[-56 \%, 51 \%]$} & {$[-66 \%, 92 \%]$} \\
\hline HDP, $2^{\text {nd }}$ Order FE & {$[<-99 \%,>1000 \%]$} & {$[-29 \%,>1000 \%]$} & {$[<-99 \%,>1000 \%]$} \\
HDP, $2^{\text {nd }}$ Order Full CMS & {$[-5 \%, 327 \%]$} & {$[-5 \%, 799 \%]$} & {$[<-99 \%,>1000 \%]$} \\
HDP, $2^{\text {nd }}$ Order Reduced CMS & {$[-19 \%, 800 \%]$} & {$[-19 \%, 800 \%]$} & {$[<-99 \%,>1000 \%]$} \\
HDP, $2^{\text {nd }}$ Order Eigenvalues & {$[<-99 \%, 153 \%]$} & {$[-40 \%, 57 \%]$} & {$[-52 \%, 83 \%]$} \\
\hline HDP, $3^{\text {rd }}$ Order FE & {$[<-99 \%,>1000 \%]$} & {$[<-99 \%,>1000 \%]$} & {$[<-99 \%,>1000 \%]$} \\
HDP, $3^{\text {rd }}$ Order Full CMS & {$[-13 \%, 14 \%]$} & {$[-13 \%, 14 \%]$} & {$[<-99 \%,>1000 \%]$} \\
HDP, $3^{\text {rd }}$ Order Reduced CMS & {$[-32 \%, 35 \%]$} & {$[-32 \%, 35 \%]$} & {$[<-99 \%,>1000 \%]$} \\
HDP, $3^{\text {rd }}$ Order Eigenvalues & {$[<-99 \%, 182 \%]$} & {$[-42 \%, 52 \%]$} & {$[-61 \%, 95 \%]$} \\
\hline
\end{tabular}

Table 2: Range of parameterizations with less than $1 \%$ error for the fifth mode. The shading of the table is based upon the range in both the positive and negative directions, with light shades having larger ranges than dark shades.

\begin{tabular}{|lrrr|}
\hline Method & $\begin{array}{r}\text { Middle Section's } \\
\text { Density, } \rho_{2}\end{array}$ & $\begin{array}{r}\text { Middle Section's } \\
\text { Location, } \ell\end{array}$ & $\begin{array}{r}\text { Middle Section's } \\
\text { Length, } W\end{array}$ \\
\hline FDP, $2^{\text {nd }}$ Order CMS & {$[-72 \%, 67 \%]$} & {$[-15 \%, 12 \%]$} & {$[-34 \%, 21 \%]$} \\
FDP, $2^{\text {nd }}$ Order Eigenvalues & {$[-54 \%, 69 \%]$} & {$[-15 \%, 17 \%]$} & {$[-85 \%, 30 \%]$} \\
\hline FDP, $4^{\text {th }}$ Order CMS & {$[-87 \%, 78 \%]$} & {$[-21 \%, 17 \%]$} & {$[-48 \%, 28 \%]$} \\
FDP, $4^{\text {th }}$ Order Eigenvalues & {$[-73 \%, 94 \%]$} & {$[-18 \%, 26 \%]$} & {$[-51 \%, 35 \%]$} \\
\hline HDP, $2^{\text {nd }}$ Order FE & {$[<-99 \%,>1000 \%]$} & {$[-5 \%, 3 \%]$} & {$[-3 \%, 3 \%]$} \\
HDP, $2^{\text {nd }}$ Order Full CMS & {$[-6 \%, 67 \%]$} & {$[-2 \%, 2 \%]$} & {$[-33 \%, 2 \%]$} \\
HDP, $2^{\text {nd }}$ Order Reduced CMS & {$[-19 \%, 68 \%]$} & {$[-9 \%, 8 \%]$} & {$[-33 \%, 10 \%]$} \\
HDP, $2^{\text {nd }}$ Order Eigenvalues & {$[<-99 \%, 154 \%]$} & {$[-12 \%, 14 \%]$} & {$[-82 \%, 29 \%]$} \\
\hline HDP, $3^{\text {rd }}$ Order FE & {$[<-99 \%,>1000 \%]$} & {$[-4 \%, 4 \%]$} & {$[-6 \%, 6 \%]$} \\
HDP, $3^{\text {rd }}$ Order Full CMS & {$[-13 \%, 14 \%]$} & {$[-5 \%, 4 \%]$} & {$[-5 \%, 5 \%]$} \\
HDP, $3^{\text {4d }}$ Order Reduced CMS & {$[-32 \%, 36 \%]$} & {$[-13 \%, 12 \%]$} & {$[-30 \%, 21 \%]$} \\
HDP, $3^{\text {rd }}$ Order Eigenvalues & {$[-64 \%, 78 \%]$} & {$[-20 \%, 17 \%]$} & {$[-32 \% 45 \%]$} \\
\hline
\end{tabular}

Table 3: Range of parameterizations with less than $1 \%$ error for the fifth mode. The shading of the table is based upon the range in both the positive and negative directions, with light shades having larger ranges than dark shades.

the full CMS parameterizations consistently are unable to predict a large range of values for each parameter except for $E_{2}$. Both the reduced CMS and eigenvalue parameterizations consistently are able to predict a significant range of responses of the system, with the exception of $\ell$; however, none of the methods investigated are able to predict a large range of the responses for $\ell$, but the fourth order FDPs and the third order eigenvalue HDP are able to predict the fifth natural frequency as $\ell$ is varied with less than $1 \%$ error for a range of approximately $\pm 20 \%$ of the nominal value of $\ell$. 
Returning to the question posed in $\S 4.2$ regarding what order parameterization is necessary, several conclusions are able to be made. For terms that enter into the FE matrices linearly, such as $E, \rho$, and $B$, a second order expansion is sufficient to parameterize these variables with a FE HDP. However, for terms that enter into the FE matrices in a nonlinear manner, the higher order FE HDPs are more accurate. As the CMS and eigenvalue PROMs have nonlinear relationships with the model parameters, it generally is found that the third order HDPs are more accurate than the second order HDPs, with the exception of the full CMS HDPs. The general conclusion from this research is that HDPs are accurate for the types of perturbations seen in parameter or sensitivity studies, but not in global optimization studies. Future work to expand the domain of validity for HDPs is focused on the construction of meta-models from several single point HDPs (Bonney et al., 2015).

\section{Multivariate Parameterization}

In the previous sections, a Taylor series expansion for a single variable is used to construct the PROMs. To construct a PROM for multiple variables, a multivariate Taylor series expansion is based on the same principles with the inclusion of cross-term derivatives. Given a variable vector $\mathbf{x}$ with nominal values $\mathbf{x}_{0}$ and a perturbation $\triangle \mathbf{x}$, the Taylor series expansion of $\mathbf{A}\left(\mathbf{x}_{0}+\triangle \mathbf{x}\right)$ is

$$
\mathbb{A}\left(\mathbf{x}_{0}+\triangle \mathbf{x}\right)=\mathbf{A}\left(\mathbf{x}_{0}\right)+\mathcal{D} \mathbf{A}\left(\mathbf{x}_{0}\right)(\triangle \mathbf{x})+\frac{1}{2}(\triangle \mathbf{x})^{T} \mathcal{H} \mathbf{A}\left(\mathbf{x}_{0}\right)(\triangle \mathbf{x})+\ldots
$$

with matrix of first derivative operators $\mathcal{D}$ and Hessian matrix $\mathcal{H}=\mathcal{D}^{2}$ of second derivative operators. With this formalized structure, generation of multivariate PROMs can be automated as only the derivative matrices (such as $\mathcal{D}$ and $\mathcal{H}$ ) are needed. These sets of derivatives are calculated in the same manner as for the single variable parameterization. Because the example application is a simple stepped beam, it is straightforward to automate the generation of all of the approximately 15,000 models necessary ${ }^{4}$ for the FDPs ; for a real application, though, this is not the case generally.

After the fourth order FDPs and third order HDPs are constructed for six parameters $\mathbf{x}=$ $\left\{E_{2}, \rho_{2}, B_{2}, H_{2}, W, \ell\right\}$, the PROMs are sampled 1,000 times using a Latin hypercube method (Minasny, 2006; Stein, 1987), sampling a normally distributed population with a mean $\mathbf{x}_{0}$ of the

\footnotetext{
${ }^{4}$ This number scales with the number of meshes per dimension raised to the number of dimensions: $5^{6}$ in this case for a third order accurate FDP.
} 

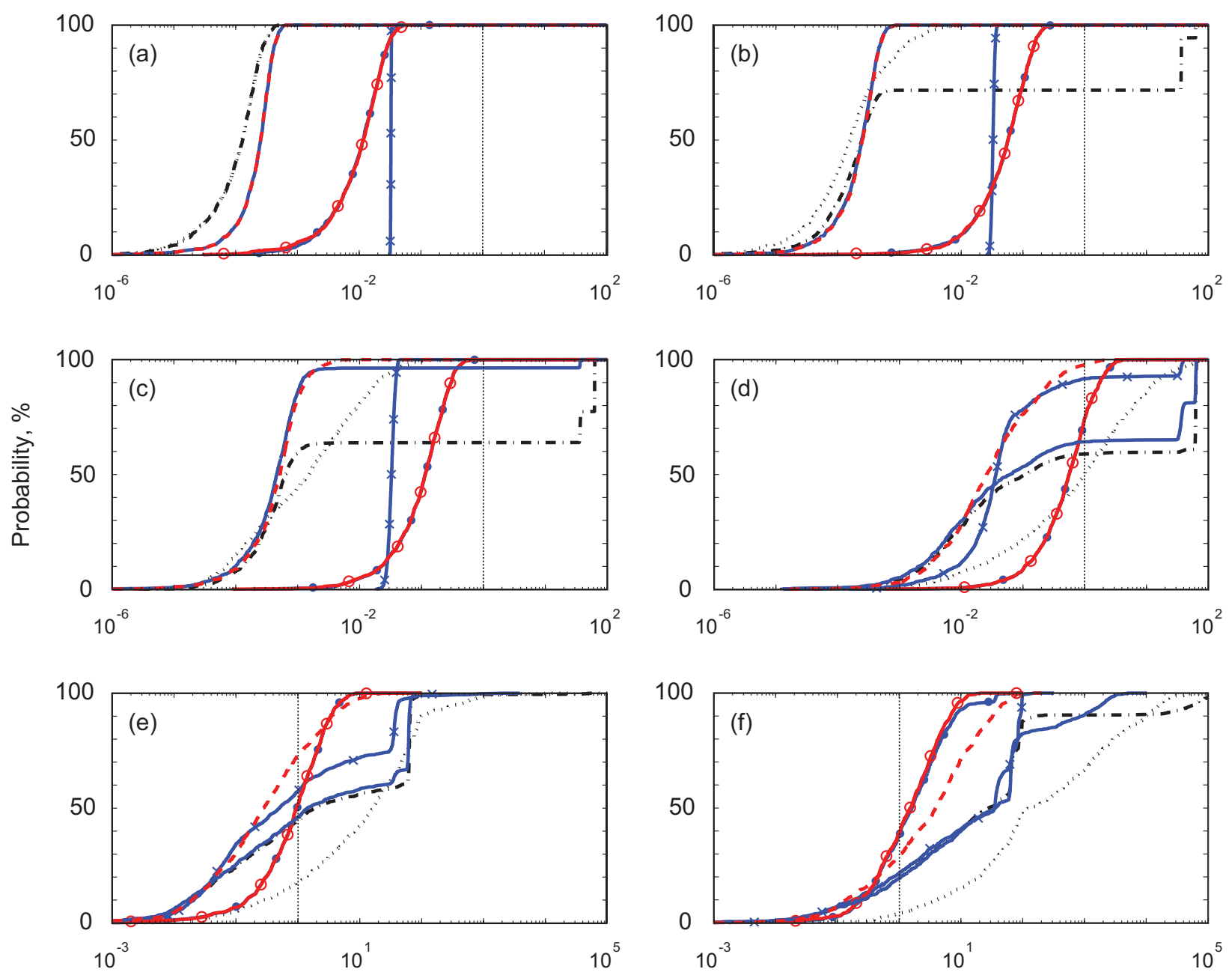

Error, \%

Figure 15: Probability function as a function of the error percentage between the predictions of the fifth natural frequency by the PROMs and the analytical solution for standard deviations of (a) $0.1 \%$, (b) $0.5 \%$, (c) $1 \%$, (d) $5 \%$, (e) $10 \%$, and (f) $20 \%$ of the mean values for the CMS FDP (blue, $-\bullet$ ), eigenvalue FDP (red, - o-), reduced CMS HDP (blue, -), reduced CMS HDP with 15 DOFs (blue, $-\times-$ ), full CMS HDP (black, - - -), eigenvalue HDP (red, $--)$, and FE HDP (black, $\cdots$ ). The thin, dashed, vertical lines indicate the $1 \%$ error threshold.

nominal variable values given in Table 1 , and six different standard deviations $(0.1 \%, 0.5 \%, 1 \%$, $5 \%, 10 \%$, and $20 \%$ of the mean values; note the different scales used for the standard deviations of $10 \%$ and $20 \%$ of the mean value), as shown in Fig. 15. Several trends are observed:

- At small perturbations, the FE HDP is the most accurate. As the standard deviation is increased above $0.1 \%$ of the mean value, though, the FE HDP transitions from being the most accurate PROM to the least accurate PROM. Similarly, the second most accurate PROM at 
the lowest standard deviation (Fig. 15(a)) is the full CMS HDP. As the standard deviation is increased, the full CMS HDP is observed to have a binomial distribution, as is evident from the large plateaus in (Fig. 15(b)-(f)).

- The predictions of the CMS FDP and eigenvalue FDP are equally accurate for all except the largest standard deviation (Fig. 15(f)), in which the eigenvalue FDP is slightly more accurate than the CMS FDP.

- The eigenvalue HDP and reduced CMS HDP are also equally accurate for small standard deviations (less than $1 \%$ of the mean value). For standard deviations greater than $1 \%$ of the mean value (Figs. 15(d)-(f)), the eigenvalue HDP is more accurate, generally, than the reduced CMS HDP.

- In comparing the eigenvalue HDP to the eigenvalue FDP, the HDP is observed to be more accurate than the FDP for standard deviations less than $20 \%$ of the mean value. This implies that for moderate changes in the design variables, the HDP is a more accurate approach.

A reduced CMS HDP with only 9 retained modes (15 DOFs including six due to interface DOFs) is also shown in Fig. 15. The constant error of $\approx 0.03 \%$ seen in Figs. 15(a)-(c) indicates that the modal truncation used for forming the reduced CMS matrices with only 15 DOFs truncated too many modes for the reduced CMS HDP to be able to reproduce exactly the analytical solution for the nominal case. For large standard deviations, it is unclear if the modal truncation effects are detrimental; for standard deviations greater than $1 \%$ of the mean value, it is difficult qualitatively to determine if the reduced CMS HDP with 36 DOFs or the reduced CMS HDP with 15 DOFs is more accurate.

To further assess the relative accuracy of the HDPs and FDPs for multivariate parameterizations to be used potentially in optimization studies, Fig. 16 shows the results of simulating 25,000 instances of the stepped beam system sampled using a Latin hypercube method from a normally distributed population with a mean of the nominal variable and a standard deviation of $50 \%$ of the mean values. Both samples with negative values and samples with center sections of the beam that extended below $x=0$ or above $x=L$ are omitted in order to keep the system well posed. Similar to the results of Fig. 15 for a standard deviation of $20 \%$ of the mean value, the eigenvalue FDP is the most accurate parameterization, followed by the CMS FDP and the eigenvalue HDP. The FE 


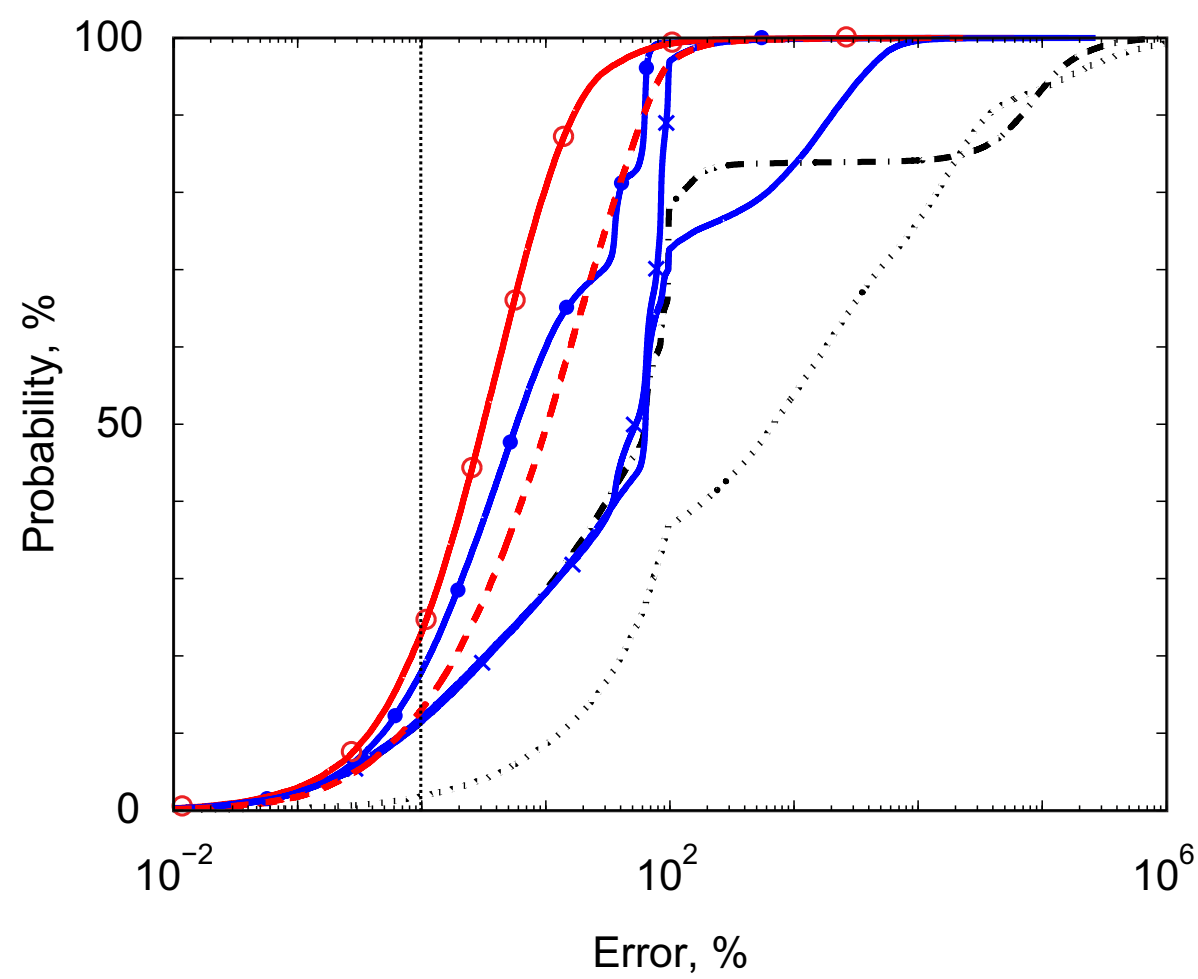

Figure 16: Probability function as a function of the error percentage between the predictions of the fifth natural frequency by the PROMs and the analytical solution for a skewed right beta distribution bounded between perturbation magnitudes of $(0,2]$ for the CMS FDP (blue, - - ), eigenvalue FDP (red, $--_{-}^{-}$), reduced CMS HDP (blue, - ), reduced CMS HDP with 15 DOFs (blue, $-\times-$ ), full CMS HDP (black, - - -), eigenvalue HDP (red, - -), and FE HDP (black, …). The thin, dashed, vertical line indicates the $1 \%$ error threshold.

HDP shows a large error (more than $60 \%$ of the samples had an error in predicting the fifth natural frequency greater than 100\%). For most samples, the eigenvalue parameterizations and the CMS FDP exhibit less than $10 \%$ error.

For each of the 31,000 samples (including the results of both Figs. 15 and 16), the error is plotted as a function of the normalized perturbation magnitude, which is defined as

$$
\mathbb{X}=\sqrt{\sum\left(\triangle \mathbf{x} / \mathbf{x}_{0}\right)^{2}}
$$

in Fig. 17. For both the full and reduced CMS HDPs, a set of samples is observed to yield large errors in the CMS HDP predictions even for small values of $\mathbb{X}$. For the full CMS HDP, this trend is exacerbated by a binomial distribution in which a cluster of samples yield errors greater than $1000 \%$ for a large range of $\mathbb{X}$. For both eigenvalue PROMs and the CMS FDP, Fig. 17 shows that the error is below $1 \%$ for most samples that are bounded by $\mathbb{X}<0.25$. For these three models, as 

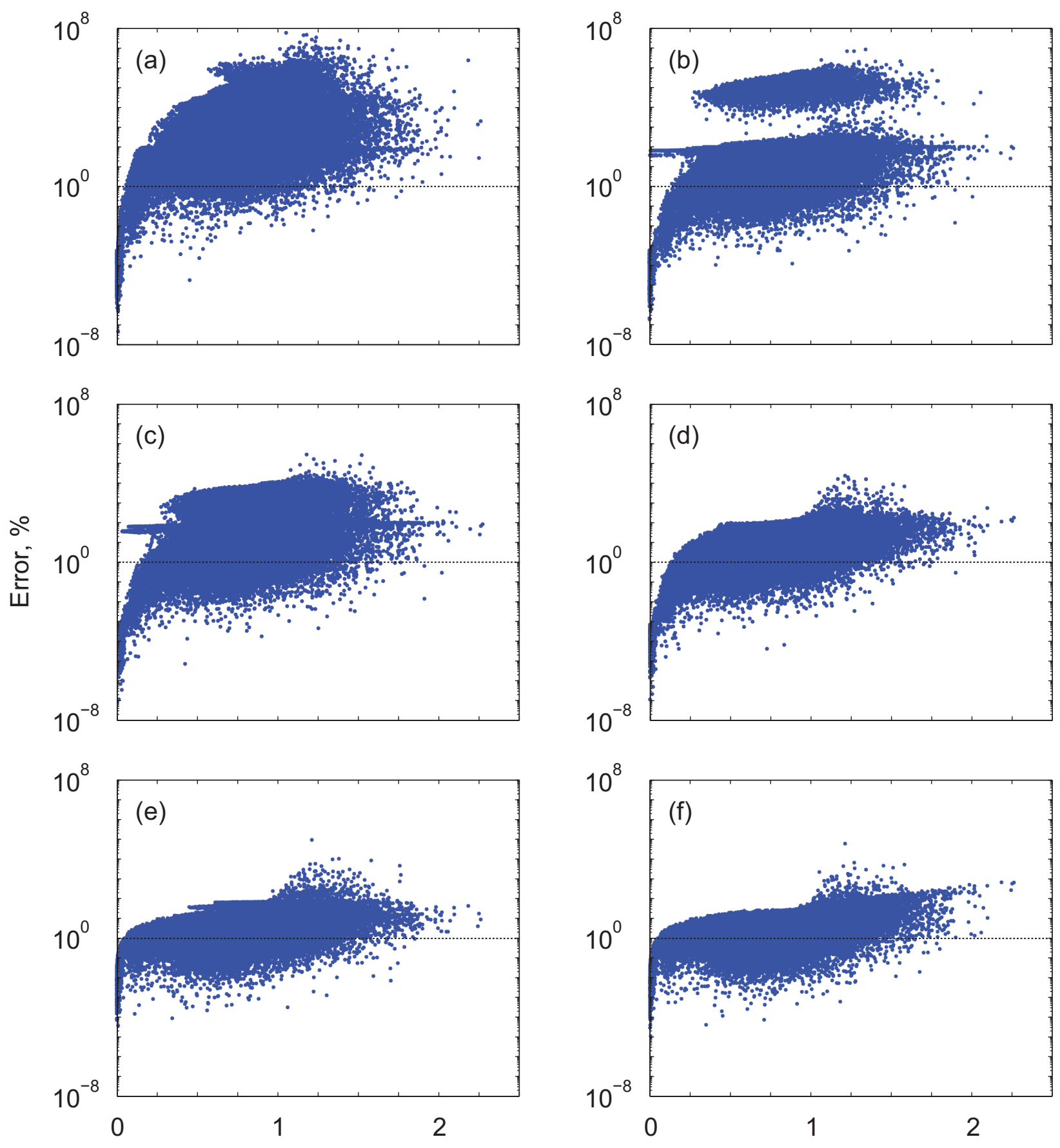

\section{Normalized Perturbation Magnitude}

Figure 17: Scatter plots of the error percentage between the predictions of the fifth natural frequency by the PROMs and the analytical solution for 31,000 samples of a skewed right beta distribution bounded between perturbation magnitudes of $(0,2]$ for the (a) FE HDP, (b) full CMS HDP, (c) reduced CMS HDP, (d) eigenvalue HDP, (e) CMS FDP, and (f) eigenvalue FDP. The thin, dashed, horizontal lines indicate the $1 \%$ error threshold. 
well, for $\mathbb{X}>0.5$ the error does not significantly increase either.

\section{Conclusions}

In this research, multiple methods for developing parameterized reduced order models are assessed. These methods are divided into two different computational techniques: finite difference based parameterizations, and hyper-dual number based parameterizations. For these two different computational techniques, the level at which the parameterization is conducted is also investigated (i.e. parameterization of the full finite element model, of a full DOF CMS model, of a reduced DOF CMS model, and of eigenvalues).

The development of the parameterizations is based upon Taylor series expansions consisting of the derivatives of the quantity being parameterized. For univariate parameterizations, finite difference based parameterizations require several meshes (a nominal mesh, and several perturbations based upon the order of the Taylor series approximations). For multivariate parameterizations, though, finite difference based techniques require a number of meshes that geometrically increases with the number of variables being parameterized. Thus, for general applications that may require an arbitrarily large number of parameterizations, the second technique (hyper-dual numbers) is introduced.

Hyper-dual numbers are based on a branch of generalized complex number theory in which $\epsilon$ equals the non-zero square root of zero. This property, substituted into a complex step expansion, allows for the exact derivatives of a quantity to be calculated based solely on a nominal mesh. As a result, multivariate parameterizations still require only a single, nominal model of the system.

The efficacy and range of validity of these two methods is investigated, and several conclusions are drawn:

- Parameterizations of CMS matrices that preserve the total number of DOFs are found to be the least accurate approach investigated for single variable parameterizations, and the parameterizations of finite element matrices are found to be the least accurate approach for multivariate parameterizations.

- In general, parameterizations of the eigenvalues are the most accurate; however:

- Parameterizations of the variables that change linearly with the system matrices or CMS 
matrices will be more accurately parameterized by a parameterization of the system matrices or CMS matrices than of the eigenvalues.

- For single-variable parameterizations of quantities that do not affect the distribution of nodes in a finite element model, finite difference parameterizations are more accurate than hyperdual number parameterizations.

- For multivariate parameterizations, the number of models required to develop a finite difference parameterization is prohibitively expensive (approximately 15,000 models necessary for the illustrative example given); whereas a hyper-dual number parameterization requires only one instance of a model.

- The order of the parameterization necessary is determined by how the variable influences the system being parameterized. For parameterizations of the finite element matrices, the matrices are linearly proportional to many of the parameters, and thus only a first order parameterization is needed. For other parameterizations, though, there is a nonlinear relationship between the system parameters and the matrices, which necessitates a higher order parameterization.

- A single mesh is sufficient for parameterizing material properties or dimensions that do not affect nodal locations (such as for the width of one-dimensional beam elements).

- For geometries that change nodal locations in the FE mesh (such as models in which the center section is moved or lengthened/shortened in the present work), a single mesh can still yield parameterizations that are accurate enough for sensitivity studies.

- Multivariate parameterizations are shown to be the most accurate for hyper-dual number parameterizations of the eigenvalues for most of the results presented. This demonstrates that the hyper-dual number framework is advantageous both in terms of reducing the number of models needed for the parameterization as well as being able to predict accurately the parameters of interest.

- For future work, an extension of the present method in which positive definiteness is guaranteed to be preserved would extend significantly the range of predictability. 
- Lastly, the computational times of the two methods are both insignificant for the example application, and will be compared in greater detail in subsequent work investigating parameterizaitons for three-dimensional applications with large numbers of DOFs. In general, a dual number calculation is twice as expensive as a purely real number calculation, and a $N$ term hyper-dual number is $2^{N}$ times as expensive as a purely real number calculation.

Future work to expand the domain of validity for HDPs to an extent that is suitable for global optimization studies is focused on the construction of meta-models from several HDPs taken at different points throughout the domain (Bonney et al., 2015).

\section{Acknowledgements}

This work was funded in part by the U.S. Department of Energy's Predictive Science Academic Alliance Program (PSAAP) Center at Stanford University. We would like to thank Rich Field, Ryan

Schultz, Remi Dingreville, Nolan McPeek-Bechtold, and Eliot Fang for feedback on and support of this research.

\section{References}

Abreu, R., Stich, D., Morales, J., 2013. On the generalization of the complex step method. Journal of Computational and Applied Mathematics 241, 84-102.

Alvin, K.F., 1997. Efficient computation of eigenvector sensitivities for structural dynamics. AIAA Journal 35, 1760-1766.

Bonney, M.S., Kammer, D.C., Brake, M.R.W., 2015. Fully parameterized reduced order models using hyper-dual numbers and component mode synthesis, in: ASME 2015 International Design Engineering Technical Conference, Boston, MA.

Brake, M.R., Barone, M.F., Segalman, D.J., 2012. Nonlinear model reduction of von Karman plates under linearized compressible fluid flow. AIAA Journal 50, 1047-1059.

Brake, M.R., Massad, J.E., Smith, R.C., Beheshti, B., Chowdhary, K., Davis, J., Wang, S., 2011. Uncertainty-enabled design of an acceleration switch, in: Proceedings of ASME 2011 International Mechanical Engineering Congress and Exposition, Denver, CO. 
Brake, M.R., Segalman, D.J., 2010. Nonlinear model reduction of von Karman plates under quasisteady fluid flow. AIAA Journal 48, 2339-2347.

Brake, M.R., Segalman, D.J., 2013. Modeling localized nonlinear constraints in continuous systems via the method of augmentation by non-smooth basis functionis. Proceedings of the Royal Society A-Mathematical Physical and Engineering Sciences 469, 1-20.

Craig, R.R., Bampton, M.C.C., 1968. Coupling of substructures for dynamic analyses. AIAA Journal 6, 1313-1319.

Field, R.V., Grigoriu, M., Emery, J.M., 2015. On the efficacy of sttochastic collocation, stochastic galerkin, and stochastic reduced order models for solving stochastic problems. Probabilistic Engineering Mechanics 41, 60-72.

Fike, J.A., Alonso, J.J., 2011. The development of hyper-dual numbers for exact second-derivative calculations, in: AIAA paper 2011-886, 49th AIAA Aerospace Sciences Meeting.

Fike, J.A., Jongsma, S., Alonso, J.J., van der Weide, E., 2011. Optimization with gradient and Hessian information calculated using hyper-dual numbers, in: AIAA paper 2011-3807, 29th AIAA Applied Aerodynamics Conference.

Fox, R.L., Kapoor, M.P., 1968. Rates of change of eigenvalues and eigenvectors. AIAA Journal 6, $2426-2429$.

Gottlieb, D., Shu, C.W., 1997. On the Gibbs phenomenon and its resolution. SIAM Review 39, $644-668$.

Grigoriu, M., 2009. Reduced order models for random functions. application to stochastic problems. Applied Mathematical Modelling 33, 161-175.

Hong, S.K., Epureanu, B.I., Castanier, M.P., 2012. Joining of components of complex structures for improved dynamic response. Journal of Sound and Vibration 331, 4285-4298.

Hong, S.K., Epureanu, B.I., Castanier, M.P., 2013. Next-generation parametric reduced-order models. Mechanical Systems and Signal Processing 37, 403-421. 
Hughes, T.J.R., 2000. The Finite Element Method; Linear Static and Dynamic Finite Element Analysis. Dover Publications, Inc.

Kammer, D.C., Krattiger, D., 2013. Propagation of uncertainty in substructured spacecraft using frequency response. AIAA Journal 51, 353-361.

Kammer, D.C., Nimityongskul, S., 2011. Propagation of uncertainty in test-analysis correlation of substructured spacecraft. Journal of Sound and Vibration 330, 1211-1224.

Karzel, H., Kist, G., 1985. Kinematic algebras and their geometries, in: Kaya, R., Plaumann, P., Strambach, K. (Eds.), Rings and Geometry, Springer. pp. 437-509.

Lai, K.L., Crassidis, J.L., 2008. Extensions of the first and second complex-step derivative approximations. Journal of Computational and Applied Mathematics 219, 276-293.

Mignolet, M.P., Soize, C., 2008. Stochastic reduced order models for uncertain geometrically nonlinear dynamical systems. Computer Methods in Applied Mechanics and Engineering 197, 3951-3963.

Minasny, B., 2006. A conditioned Latin hypercube method for sampling in the presence of ancillary information. Computers and Geosciences 32, 1378-1388.

Momin, F.N., Millwater, H.R., Osborn, R.W., Enright, M.P., 2010. A non-intrusive method to add finite element-based random variables to a probabilistic design code. Finite Elements in Analysis and Design 46, 280-287.

Nelson, R.B., 1976. Simplified calculation of eigenvector derivatives. AIAA Journal 14, 1201-1205.

Sauer, T., 1995. Computational aspects of multivariate polynomial interpolation. Advances in Computational Mathematics 3, 219-237.

Stefanou, G., 2009. The stochastic finite element method: Past, present, and future. Computer Methods in Applied Mechanics and Engineering 198, 1031-1051.

Stein, M., 1987. Large sample properties of simulations using Latin hypercube sampling. Technometrics 29, 143-151.

Study, E., 1903. Geometrie der Dynamen. B. G. Teubner. 
Voorhees, A., Millwater, H.R., Bagley, R., 2011. Complex variable methods for shape sensitivity of finite element models. Finite Elements in Analysis and Design 47, 1146-1156.

Xia, Z., Tang, J., 2013. Characterization of dynamic response of structures with uncertainty by using Gaussian processes. ASME Journal of Vibration and Acoustics 135, 051006-1-13.

Young, J.T., 2000. Finite Element Modeling Continuous Improvement. NASA. chapter Primer on the Craig-Bampton Method.

\section{Appendix A. Analytical Solution for the Stepped Beam}

The stepped beam is divided into three contiguous regions: $[0, \ell-W / 2],[\ell-W / 2, \ell+W / 2]$, and $[\ell+W / 2, L]$ that are sequentially numbered $j=1,2$, and 3 respectively. In each region, the beam is modeled as an Euler-Bernoulli beam such that in region $j$

$$
\rho_{j} A_{j} \frac{\partial^{2} w_{j}}{\partial t^{2}}+E_{j} I_{j} \frac{\partial^{4} w_{j}}{\partial x^{4}}=0
$$

Here, $E_{1}=E_{3}=E, \rho_{1}=\rho_{3}=\rho, I_{1}=I_{3}=B H^{3} / 12, A_{1}=A_{3}=B H, I_{2}=B_{2} H_{2}^{3} / 12$, and $A_{2}=B_{2} H_{2}$. The three regions are subject to the boundary conditions

$$
w_{1}(0, t)=0
$$




$$
\begin{aligned}
\left.\frac{\partial^{2} w_{1}}{\partial x^{2}}\right|_{x=0}=0 & \\
w_{1}(\ell-W / 2, t) & =w_{2}(\ell-W / 2, t) \\
\left.\frac{\partial w_{1}}{\partial x}\right|_{x=\ell-W / 2} & =\left.\frac{\partial w_{2}}{\partial x}\right|_{x=\ell-W / 2} \\
\left.E I_{1} \frac{\partial^{2} w_{1}}{\partial x^{2}}\right|_{x=\ell-W / 2} & =\left.E_{2} I_{2} \frac{\partial^{2} w_{2}}{\partial x^{2}}\right|_{x=\ell-W / 2} \\
\left.E I_{1} \frac{\partial^{3} w_{1}}{\partial x^{3}}\right|_{x=\ell-W / 2} & =\left.E_{2} I_{2} \frac{\partial^{3} w_{2}}{\partial x^{3}}\right|_{x=\ell-W / 2} \\
w_{2}(\ell+W / 2, t) & =w_{3}(\ell+W / 2, t) \\
\left.\frac{\partial w_{2}}{\partial x}\right|_{x=\ell+W / 2} & =\left.\frac{\partial w_{3}}{\partial x}\right|_{x=\ell+W / 2} \\
\left.E_{2} I_{2} \frac{\partial^{2} w_{2}}{\partial x^{2}}\right|_{x=\ell+W / 2} & =\left.E I_{3} \frac{\partial^{2} w_{3}}{\partial x^{2}}\right|_{x=\ell+W / 2} \\
\left.E_{2} I_{2} \frac{\partial^{3} w_{2}}{\partial x^{3}}\right|_{x=\ell+W / 2} & =\left.E I_{3} \frac{\partial^{3} w_{3}}{\partial x^{3}}\right|_{x=\ell+W / 2} \\
w_{3}(L, t) & =0 \\
\left.\frac{\partial^{2} w_{3}}{\partial x^{2}}\right|_{x=L} & =0 .
\end{aligned}
$$

Note that for large perturbations, the assumptions of Euler-Bernoulli beam theory break down; however, as the reference model is based on Euler-Bernoulli beam theory, it is able to provide a basis for comparison of the PROMs to the analytical solution that they are based on. Assuming a separable solution, $w_{j}$ is of the form

$$
w_{j}(x, t)=\sum_{n=1}^{N} e^{\mathrm{i} \omega_{n} t} \phi_{j n}(x),
$$

with $\mathrm{i}=\sqrt{-1}, N$ being the number of terms taken in the modal expansion solution, and

$$
\phi_{j n}(x)=A_{j n} \sin \left(\lambda_{j n} x\right)+B_{j n} \cos \left(\lambda_{j n} x\right)+C_{j n} \sinh \left(\lambda_{j n} x\right)+D_{j n} \cosh \left(\lambda_{j n} x\right) .
$$

Substitution of the modal expansion solution (Eq. A.15) into the boundary conditions (Eqs. A.2A.13), with the assumption that the natural frequencies of all three contiguous regions are the same 
(i.e. that linear vibration theory applies) and the relation that

$$
\omega_{n}=\omega_{j n}=\sqrt{\frac{E_{j} I_{j}}{\rho_{j} A_{j}}} \lambda_{j n}^{2} \quad j=1,2,3,
$$

yields the eigenvalue problem from which the natural frequencies $\omega_{n}$ and mode shapes of the system can be calculated.

\section{Appendix B. Functions of Hyper-Dual Numbers}

As discussed above, the use of hyper-dual numbers requires overloading all of the functions in an analysis code to operate on hyper-dual numbers instead of on real numbers. A hyper-dual number function takes hyper-dual numbers as inputs and produces hyper-dual numbers as outputs. If the source code for the function is available, then it is straight forward to modify the internal operations of the function to use hyper-dual numbers. However, there are often situations where the source code is unavailable, such as when using built-in functions or external third-party libraries. In these situations it is still possible to use hyper-dual numbers if the required derivatives can be computed in some other way and then combined to form the required hyper-dual output.

This is demonstrated for the solution of a matrix system and the calculation of eigenvalues and eigenvectors, both of which are required for the Craig-Bampton Component Mode Synthesis method and which typically use routines for which the source code is unavailable. The strategy in both of these cases is to derive expressions for the resulting derivatives. These expressions will involve derivatives of the inputs, which can be extracted from the hyper-dual number input. Once the resulting calculations are complete, the resulting real-valued derivatives can then be formed into the required hyper-dual number output.

Appendix B.1. Derivatives of Matrix System Solves

Consider the solution of a system

$$
\mathbf{A}(\mathbf{y}, \mathbf{x}) \mathbf{y}(\mathbf{x})=\mathbf{b}(\mathbf{y}, \mathbf{x})
$$

where both the system matrix $\mathbf{A}$ and the force vector $\mathbf{b}$ are dependent on the state variable $\mathbf{y}$ and $n$ system parameters of interest $\mathbf{x}=\left[x_{1} \ldots x_{n}\right]$. Once $\mathbf{A}$ and $\mathbf{b}$ are constructed from a particular instance of $\mathbf{y}$ and $\mathbf{x}$, the system can be linearized about this point as

$$
\mathbf{A}_{\text {lin }} \mathbf{y}=\mathbf{b}_{\text {lin }}
$$


and derivatives can be computed by several calls to the real-valued routine (Fike et al., 2011). The first derivatives of the solution of Eq. B.2, $\partial \mathbf{y} / \partial x_{i}$, with respect to the $i^{\text {th }}$ system parameter, can be computed by solving

$$
\mathbf{A}_{\operatorname{lin}} \frac{\partial \mathbf{y}}{\partial x_{i}}=\frac{\partial \mathbf{b}_{\operatorname{lin}}}{\partial x_{i}}-\frac{\partial \mathbf{A}_{\operatorname{lin}}}{\partial x_{i}} \mathbf{y} .
$$

Second derivatives with respect to the $i^{\text {th }}$ and $j^{\text {th }}$ system variables, subsequently, are found as the solution of

$$
\mathbf{A}_{\operatorname{lin}} \frac{\partial^{2} \mathbf{y}}{\partial x_{i} \partial x_{j}}=\frac{\partial^{2} \mathbf{b}_{\operatorname{lin}}}{\partial x_{i} \partial x_{j}}-\frac{\partial^{2} \mathbf{A}_{\operatorname{lin}}}{\partial x_{i} \partial x_{j}} \mathbf{y}-\frac{\partial \mathbf{A}_{\operatorname{lin}}}{\partial x_{i}} \frac{\partial \mathbf{y}}{\partial x_{j}}-\frac{\partial \mathbf{A}_{\operatorname{lin}}}{\partial x_{j}} \frac{\partial \mathbf{y}}{\partial x_{i}}
$$

and third derivatives with respect to the $i^{\text {th }}, j^{\text {th }}$, and $k^{\text {th }}$ system variables are calculated by solving

$$
\begin{aligned}
\mathbf{A}_{\operatorname{lin}} \frac{\partial^{3} \mathbf{y}}{\partial x_{i} \partial x_{j} \partial x_{k}}=\frac{\partial^{3} \mathbf{b}_{\operatorname{lin}}}{\partial x_{i} \partial x_{j} \partial x_{k}}-\frac{\partial^{3} \mathbf{A}_{\operatorname{lin}}}{\partial x_{i} \partial x_{j} \partial x_{k}} \mathbf{y}-\frac{\partial^{2} \mathbf{A}_{\operatorname{lin}}}{\partial x_{i} \partial x_{j}} \frac{\partial \mathbf{y}}{\partial x_{k}}-\frac{\partial^{2} \mathbf{A}_{\operatorname{lin}}}{\partial x_{i} \partial x_{k}} \frac{\partial \mathbf{y}}{\partial x_{j}} \\
\quad-\frac{\partial^{2} \mathbf{A}_{\operatorname{lin}}}{\partial x_{j} \partial x_{k}} \frac{\partial \mathbf{y}}{\partial x_{i}}-\frac{\partial \mathbf{A}_{\operatorname{lin}}}{\partial x_{i}} \frac{\partial^{2} \mathbf{y}}{\partial x_{j} \partial x_{k}}-\frac{\partial \mathbf{A}_{\operatorname{lin}}}{\partial x_{j}} \frac{\partial^{2} \mathbf{y}}{\partial x_{i} \partial x_{k}}-\frac{\partial \mathbf{A}_{\operatorname{lin}}}{\partial x_{k}} \frac{\partial^{2} \mathbf{y}}{\partial x_{i} \partial x_{j}} .
\end{aligned}
$$

The derivatives of $\mathbf{A}_{\text {lin }}$ and $\mathbf{b}_{\text {lin }}$ that are required in the above expressions can be extracted from the hyper-dual number versions of these inputs. Once the values and derivatives of $\mathbf{y}$ are computed they can then be used to form the required hyper-dual number output.

\section{Appendix B.2. Derivatives of Eigenvalues and Eigenvectors}

Using hyper-dual numbers to compute derivatives for the parameterization of eigenvalues or CMS matrices requires a hyper-dual version of the eigenvalue calculation routine. As with the solution of a linear system, the effect of using hyper-dual numbers can be recreated without modifying the real-valued eigenvalue solver. To create a parameterization of the $\ell^{\text {th }}$ eigenvalue $\lambda_{\ell}$ and eigenvector $\phi_{\ell}$, it is necessary to calculate the derivatives of the eigenvalues and eigenvectors with respect to the model parameters $\mathbf{x}$. Eigenvalues and eigenvectors are solutions of

$$
\left(\mathbf{K}(\mathbf{x})-\lambda_{\ell} \mathbf{M}(\mathbf{x})\right) \phi_{\ell}=\mathbf{F}_{\ell}(\mathbf{x}) \phi_{\ell}=0
$$

with $\mathbf{F}_{\ell}=\left(\mathbf{K}-\lambda_{\ell} \mathbf{M}\right)$. This equation can be differentiated with respect to the $i^{\text {th }}$ system parameter to give

$$
\frac{\partial \mathbf{F}_{\ell}}{\partial x_{i}} \phi_{\ell}+\mathbf{F}_{\ell} \frac{\partial \phi_{\ell}}{\partial x_{i}}=0
$$


Pre-multiplying this equation by the transpose of the eigenvector, and making use of the fact that $\mathbf{F}_{\ell} \phi_{\ell}=0$, yields

$$
\boldsymbol{\phi}_{\ell}^{T} \frac{\partial \mathbf{F}_{\ell}}{\partial x_{i}} \phi_{\ell}=\phi_{\ell}^{T}\left(\frac{\partial \mathbf{K}}{\partial x_{i}}-\lambda_{\ell} \frac{\partial \mathbf{M}}{\partial x_{i}}-\frac{\partial \lambda_{\ell}}{\partial x_{i}} \mathbf{M}\right) \phi_{\ell}
$$

The eigenvectors are orthonormal with respect to $\mathbf{M}$, thus $\boldsymbol{\phi}_{\ell}^{T} \mathbf{M} \phi_{\ell}=1$. Rearranging (B.8) gives the first derivative of the eigenvalue

$$
\frac{\partial \lambda_{\ell}}{\partial x_{i}}=\phi_{\ell}^{T}\left(\frac{\partial \mathbf{K}}{\partial x_{i}}-\lambda_{\ell} \frac{\partial \mathbf{M}}{\partial x_{i}}\right) \phi_{\ell}
$$

There are several methods for computing the first derivatives of eigenvectors, as summarized by Alvin (Alvin, 1997). The method of Nelson (Nelson, 1976) is exact but can be computationally expensive since it involves solving a linear system for each derivative. The modal superposition method (Fox and Kapoor, 1968) represents the derivative of an eigenvector as a superposition of the other eigenvectors and is less computationally intensive than Nelson's method.

Nelson's method for computing the first derivative of an eigenvector with respect to the $i^{\text {th }}$ system parameter is to represent it as a sum of two terms

$$
\frac{\partial \phi_{\ell}}{\partial x_{i}}=\boldsymbol{z}_{i}+c_{i} \phi_{\ell}
$$

The quantity $\mathbf{z}_{i}$ is found by solving (B.7) with $\partial \phi_{\ell} / \partial x_{i}$ replaced by $\mathbf{z}_{i}$,

$$
\mathbf{F}_{\ell} \mathbf{z}_{i}=-\frac{\partial \mathbf{F}_{\ell}}{\partial x_{i}} \phi_{\ell}
$$

The contribution of $\phi_{\ell}$ is added back in by multiplying by $c_{i}$, which is found by differentiating the orthonormalization equation

$$
\phi_{j}^{T} \mathbf{M} \phi_{k}=\delta_{j k}
$$

where $\delta_{j k}$ is the Kronecker delta function, and $\ell=j, k$. This yields the first derivative of the orthonormalization equation for $j=k=\ell$ (Alvin, 1997)

$$
\phi_{\ell}^{T} \frac{\partial \mathbf{M}}{\partial x_{i}} \phi_{\ell}+2 \phi_{\ell}^{T} \mathbf{M} \frac{\partial \phi_{\ell}}{\partial x_{i}}=0
$$

Substituting Eq. B.10 into this equation produces

$$
\phi_{\ell}^{T} \frac{\partial \mathbf{M}}{\partial x_{i}} \phi_{\ell}+2 \phi_{\ell}^{T} \mathbf{M}\left(\mathbf{z}_{i}+c_{i} \phi_{\ell}\right)=0
$$


with

$$
c_{i}=-\frac{1}{2} \phi_{\ell}^{T} \frac{\partial \mathbf{M}}{\partial x_{i}} \phi_{\ell}-\phi_{\ell}^{T} \mathbf{M} \mathbf{z}_{i}
$$

Figure B.1 shows the first derivative of the second eigenvector with respect to the cross-sectional height $\left(x_{1}=H_{2}\right.$ in the example of $\left.\S 5\right)$ computed using finite differences, Nelson's method, and modal superposition. Nelson's method and the finite difference calculation are in good agreement. The first derivative of the eigenvector has sharp corners where the individual components join together. Modal superposition requires the use of all eigenvectors in order to be exact. Using a smaller subset results in an approximation. In Fig. B.1, 20 modes are used for the modal superposition method, which does not capture the sharp corners, and exhibits the Gibbs phenomenon (Gottlieb and Shu, 1997), as would be expected of a method relying on a finite summation of modes. When the application is CMS, only a few eigenvectors are kept, so the computational cost of Nelson's method is not expected to be an issue and is preferred over modal superposition.

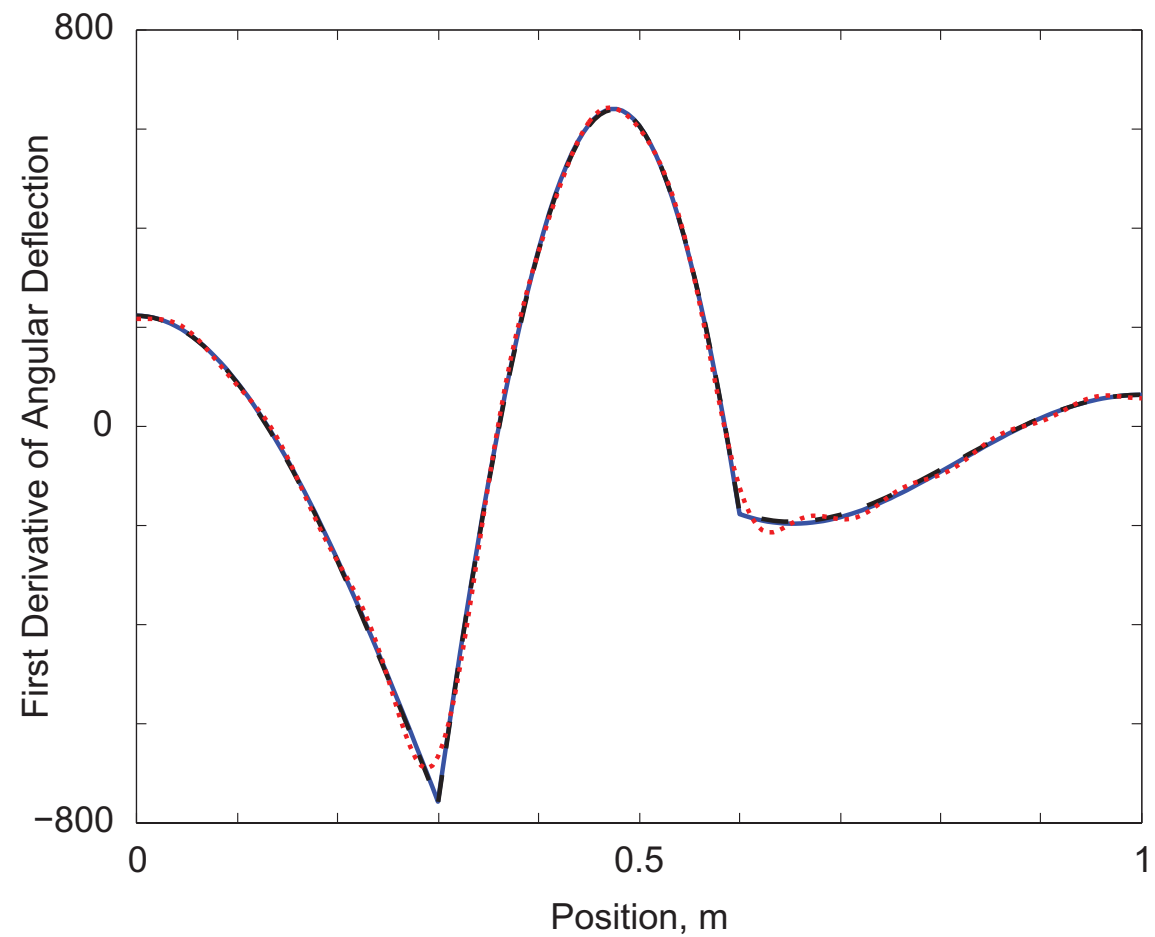

Figure B.1: A comparison of the finite difference method (black, --), modal superposition method (Fox and Kapoor, 1968) (red, ...), and Nelson's method (Nelson, 1976) (blue, - ) for computing derivatives of eigenvectors; the system and its parameters are described in $\S 5$. The results shown are for the first derivative of the second bending mode taken with respect to the cross-sectional height.

Expressions for second and third derivatives can be derived following a similar procedure. The 
second derivative of an eigenvalue with respect to the $i^{\text {th }}$ and $j^{\text {th }}$ system parameters can be computed as

$$
\frac{\partial^{2} \lambda_{\ell}}{\partial x_{i} \partial x_{j}}=\phi_{\ell}^{T}\left(\frac{\partial^{2} \mathbf{K}}{\partial x_{i} \partial x_{j}}-\frac{\partial \lambda_{\ell}}{\partial x_{i}} \frac{\partial \mathbf{M}}{\partial x_{j}}-\frac{\partial \lambda_{\ell}}{\partial x_{j}} \frac{\partial \mathbf{M}}{\partial x_{i}}-\lambda_{\ell} \frac{\partial^{2} \mathbf{M}}{\partial x_{i} \partial x_{j}}\right) \phi_{\ell}+\phi_{\ell}^{T} \frac{\partial \mathbf{F}_{\ell}}{\partial x_{i}} \frac{\partial \boldsymbol{\phi}_{\ell}}{\partial x_{j}}+\boldsymbol{\phi}_{\ell}^{T} \frac{\partial \mathbf{F}_{\ell}}{\partial x_{j}} \frac{\partial \boldsymbol{\phi}_{\ell}}{\partial x_{i}},
$$

and the second derivative of the corresponding eigenvector is

$$
\frac{\partial^{2} \boldsymbol{\phi}_{\ell}}{\partial x_{i} \partial x_{j}}=\mathbf{z}_{i j}+c_{i j} \phi_{\ell}
$$

where

$$
c_{i j}=-\frac{1}{2} \boldsymbol{\phi}_{\ell}^{T} \frac{\partial^{2} \mathbf{M}}{\partial x_{i} \partial x_{j}} \boldsymbol{\phi}_{\ell}-\boldsymbol{\phi}_{\ell}^{T} \frac{\partial \mathbf{M}}{\partial x_{i}} \frac{\partial \phi_{\ell}}{\partial x_{j}}-\boldsymbol{\phi}_{\ell}^{T} \frac{\partial \mathbf{M}}{\partial x_{j}} \frac{\partial \phi_{\ell}}{\partial x_{i}}-\frac{\partial \phi_{\ell}^{T}}{\partial x_{j}} \mathbf{M} \frac{\partial \phi_{\ell}}{\partial x_{i}}-\boldsymbol{\phi}_{\ell}^{T} \mathbf{M z}_{i j}
$$

The term $\mathbf{z}_{i j}$ is found by solving

$$
\mathbf{F}_{\ell} \mathbf{z}_{i j}=-\frac{\partial^{2} \mathbf{F}_{\ell}}{\partial x_{i} \partial x_{j}} \boldsymbol{\phi}_{\ell}-\frac{\partial \mathbf{F}_{\ell}}{\partial x_{i}} \frac{\partial \boldsymbol{\phi}_{\ell}}{\partial x_{j}}-\frac{\partial \mathbf{F}_{\ell}}{\partial x_{j}} \frac{\partial \boldsymbol{\phi}_{\ell}}{\partial x_{i}}
$$

Similarly, the expression for the third derivative of an eigenvalue with respect to the $i^{\text {th }}, j^{\text {th }}$, and $k^{\text {th }}$ system parameters is

$$
\begin{gathered}
\frac{\partial^{3} \lambda_{\ell}}{\partial x_{i} \partial x_{j} \partial x_{k}}=\boldsymbol{\phi}_{\ell}^{T}\left(\frac{\partial^{3} \mathbf{K}}{\partial x_{i} \partial x_{j} \partial x_{k}}-\frac{\partial^{2} \lambda_{\ell}}{\partial x_{j} \partial x_{k}} \frac{\partial \mathbf{M}}{\partial x_{i}}-\frac{\partial^{2} \lambda_{\ell}}{\partial x_{i} \partial x_{k}} \frac{\partial \mathbf{M}}{\partial x_{j}}-\frac{\partial^{2} \lambda_{\ell}}{\partial x_{i} \partial x_{j}} \frac{\partial \mathbf{M}}{\partial x_{k}}-\frac{\partial \lambda_{\ell}}{\partial x_{i}} \frac{\partial^{2} \mathbf{M}}{\partial x_{j} \partial x_{k}}\right. \\
\left.-\frac{\partial \lambda_{\ell}}{\partial x_{j}} \frac{\partial^{2} \mathbf{M}}{\partial x_{i} \partial x_{k}}-\frac{\partial \lambda_{\ell}}{\partial x_{k}} \frac{\partial^{2} \mathbf{M}}{\partial x_{i} \partial x_{j}}-\lambda_{\ell} \frac{\partial^{3} \mathbf{M}}{\partial x_{i} \partial x_{j} \partial x_{k}}\right) \boldsymbol{\phi}_{\ell}+\boldsymbol{\phi}_{\ell}^{T} \frac{\partial^{2} \mathbf{F}_{\ell}}{\partial x_{j} \partial x_{k}} \frac{\partial \boldsymbol{\phi}_{\ell}}{\partial x_{i}}+\boldsymbol{\phi}_{\ell}^{T} \frac{\partial^{2} \mathbf{F}_{\ell}}{\partial x_{i} \partial x_{k}} \frac{\partial \boldsymbol{\phi}_{\ell}}{\partial x_{j}} \\
+\boldsymbol{\phi}_{\ell}^{T} \frac{\partial^{2} \mathbf{F}_{\ell}}{\partial x_{i} \partial x_{j}} \frac{\partial \boldsymbol{\phi}_{\ell}}{\partial x_{k}}+\boldsymbol{\phi}_{\ell}^{T} \frac{\partial \mathbf{F}_{\ell}}{\partial x_{i}} \frac{\partial^{2} \boldsymbol{\phi}_{\ell}}{\partial x_{j} \partial x_{k}}+\boldsymbol{\phi}_{\ell}^{T} \frac{\partial \mathbf{F}_{\ell}}{\partial x_{j}} \frac{\partial^{2} \boldsymbol{\phi}_{\ell}}{\partial x_{i} \partial x_{k}}+\boldsymbol{\phi}_{\ell}^{T} \frac{\partial \mathbf{F}_{\ell}}{\partial x_{k}} \frac{\partial^{2} \boldsymbol{\phi}_{\ell}}{\partial x_{i} \partial x_{j}},
\end{gathered}
$$

with derivatives of the corresponding eigenvectors

$$
\frac{\partial^{3} \boldsymbol{\phi}_{\ell}}{\partial x_{i} \partial x_{j} \partial x_{k}}=\mathbf{z}_{i j k}+c_{i j k} \boldsymbol{\phi}_{\ell}
$$

where

$$
\begin{array}{r}
c_{i j k}=-\frac{1}{2} \boldsymbol{\phi}_{\ell}^{T} \frac{\partial^{3} \mathbf{M}}{\partial x_{i} \partial x_{j} \partial x_{k}} \boldsymbol{\phi}_{\ell}-\boldsymbol{\phi}_{\ell}^{T} \frac{\partial^{2} \mathbf{M}}{\partial x_{i} \partial x_{j}} \frac{\partial \boldsymbol{\phi}_{\ell}}{\partial x_{k}}-\boldsymbol{\phi}_{\ell}^{T} \frac{\partial^{2} \mathbf{M}}{\partial x_{i} \partial x_{k}} \frac{\partial \boldsymbol{\phi}_{\ell}}{\partial x_{j}}-\boldsymbol{\phi}_{\ell}^{T} \frac{\partial^{2} \mathbf{M}}{\partial x_{j} \partial x_{k}} \frac{\partial \boldsymbol{\phi}_{\ell}}{\partial x_{i}}-\frac{\partial \boldsymbol{\phi}_{\ell}^{T}}{\partial x_{k}} \frac{\partial \mathbf{M}}{\partial x_{i}} \frac{\partial \boldsymbol{\phi}_{\ell}}{\partial x_{j}} \\
-\frac{\partial \boldsymbol{\phi}_{\ell}^{T}}{\partial x_{k}} \frac{\partial \mathbf{M}}{\partial x_{j}} \frac{\partial \boldsymbol{\phi}_{\ell}}{\partial x_{i}}-\frac{\partial \boldsymbol{\phi}_{\ell}^{T}}{\partial x_{k}} \mathbf{M} \frac{\partial^{2} \boldsymbol{\phi}_{\ell}}{\partial x_{i} \partial x_{j}}-\frac{\partial \boldsymbol{\phi}_{\ell}^{T}}{\partial x_{j}} \frac{\partial \mathbf{M}}{\partial x_{k}} \frac{\partial \boldsymbol{\phi}_{\ell}}{\partial x_{i}}-\frac{\partial^{2} \boldsymbol{\phi}_{\ell}^{T}}{\partial x_{j} \partial x_{k}} \mathbf{M} \frac{\partial \boldsymbol{\phi}_{\ell}}{\partial x_{i}}-\frac{\partial \boldsymbol{\phi}_{\ell}^{T}}{\partial x_{j}} \mathbf{M} \frac{\partial^{2} \boldsymbol{\phi}_{\ell}}{\partial x_{i} \partial x_{k}} \\
-\boldsymbol{\phi}_{\ell}^{T} \frac{\partial \mathbf{M}}{\partial x_{i}} \frac{\partial^{2} \boldsymbol{\phi}_{\ell}}{\partial x_{j} \partial x_{k}}-\boldsymbol{\phi}_{\ell}^{T} \frac{\partial \mathbf{M}}{\partial x_{j}} \frac{\partial^{2} \boldsymbol{\phi}_{\ell}}{\partial x_{i} \partial x_{k}}-\boldsymbol{\phi}_{\ell}^{T} \frac{\partial \mathbf{M}}{\partial x_{k}} \frac{\partial^{2} \boldsymbol{\phi}_{\ell}}{\partial x_{i} \partial x_{j}}-\boldsymbol{\phi}_{\ell}^{T} \mathbf{M} \mathbf{z}_{i j k}, \quad \text { (B.22) }
\end{array}
$$


and $\mathbf{z}_{i j k}$ is from the solution of

$$
\begin{aligned}
\mathbf{F}_{\ell} \mathbf{z}_{i j k}=-\frac{\partial^{3} \mathbf{F}_{\ell}}{\partial x_{i} \partial x_{j} \partial x_{k}} \boldsymbol{\phi}_{\ell}-\frac{\partial^{2} \mathbf{F}_{\ell}}{\partial x_{j} \partial x_{k}} \frac{\partial \boldsymbol{\phi}_{\ell}}{\partial x_{i}}- & \frac{\partial^{2} \mathbf{F}_{\ell}}{\partial x_{i} \partial x_{k}} \frac{\partial \boldsymbol{\phi}_{\ell}}{\partial x_{j}}-\frac{\partial^{2} \mathbf{F}_{\ell}}{\partial x_{i} \partial x_{j}} \frac{\partial \boldsymbol{\phi}_{\ell}}{\partial x_{k}} \\
& -\frac{\partial \mathbf{F}_{\ell}}{\partial x_{i}} \frac{\partial^{2} \boldsymbol{\phi}_{\ell}}{\partial x_{j} \partial x_{k}}-\frac{\partial \mathbf{F}_{\ell}}{\partial x_{j}} \frac{\partial^{2} \boldsymbol{\phi}_{\ell}}{\partial x_{i} \partial x_{k}}-\frac{\partial \mathbf{F}_{\ell}}{\partial x_{k}} \frac{\partial^{2} \boldsymbol{\phi}_{\ell}}{\partial x_{i} \partial x_{j}} .
\end{aligned}
$$

The derivatives of $\mathbf{M}$ and $\mathbf{K}$ that are required in the above expressions can be extracted from the hyper-dual number versions of these inputs. Once the values and derivatives of $\lambda_{\ell}$ and $\phi_{\ell}$ are computed they can then be used to form the required hyper-dual number outputs. 